\title{
Iterative schemes for surfactant transport in porous media
}

\author{
Davide Illiano ${ }^{1} \cdot$ Iuliu Sorin Pop ${ }^{1,2} \cdot$ Florin Adrian Radu ${ }^{1}$
}

Received: 31 May 2019 / Accepted: 21 February 2020 / Published online: 14 June 2020

(c) The Author(s) 2020

\begin{abstract}
In this work, we consider the transport of a surfactant in variably saturated porous media. The water flow is modelled by the Richards equations and it is fully coupled with the transport equation for the surfactant. Three linearization techniques are discussed: the Newton method, the modified Picard, and the L-scheme. Based on these, monolithic and splitting schemes are proposed and their convergence is analyzed. The performance of these schemes is illustrated on five numerical examples. For these examples, the number of iterations and the condition numbers of the linear systems emerging in each iteration are presented.
\end{abstract}

Keywords Richards equation - Reactive transport - Linearization schemes · L-scheme · Modified Picard . Newton method $\cdot$ Splitting solvers

\section{Introduction}

Many societally relevant applications are involving multiphase flow and multicomponent reactive transport in porous media. Examples in this sense appear in the enhanced oil recovery, geological $\mathrm{CO}_{2}$ storage, diffusion of medical agents into the human body, or water or soil pollution. In many situations like these, experimental results are difficult and expensive to obtain, therefore numerical simulations become a key technology. Together with laboratory experiments and field data, they provide the key tools in understanding such complex phenomena. The mathematical models for problems as mentioned above are (fully or partially) coupled, nonlinear, possible degenerate partial differential equations. In most cases, deriving explicit solutions is not possible, whereas developing appropriate algorithms

Iuliu Sorin Pop

sorin.pop@uhasselt.be; Iuliu.Pop@uib.no

Davide Illiano

Davide.Illiano@uib.no

Florin Adrian Radu

Florin.Radu@uib.no

1 Department of Mathematics, University of Bergen, Allegaten 41, Bergen, Norway

2 Faculty of Sciences, University of Hasselt, Agoralaan Building D, BE 3590, Diepenbeek, Belgium for finding numerical solutions is a challenge in itself. Here we investigate robust and efficient methods for solving the nonlinear problems obtained after performing an implicit time discretization. The focus is on iterative, splitting, or monolithic schemes for fully coupled flow and transport.

Of particular interest here is a special case of multiphase, reactive flow in porous media, namely the surfactant transport in soil [2, 19, 23, 25, 27, 33]. Surfactants, which are usually organic compounds, are commonly used for actively combating soil and water pollution $[11,12,16,38$, 43]. They contain both hydrophobic and hydrophilic groups and are dissolved in the water phase, being transported by diffusion and convection. Typically, the surfactants are employed in soil regions near the surface (vadose zone), where water and air are present in the pores. Consequently, the outcoming mathematical model accounts the transport of at least one species (the surfactant, but often also the contaminant) in a variably saturated porous medium. Whereas the dependence of the species transported from the flow is obvious, one can encounter the reverse dependence as well when surfactants are affecting the interfacial tension between water and air, leading to a dependency of the water flow on the concentration of surfactant. In other words, one has to cope with a fully coupled flow and transport problem, and not only with a one-way coupling, i.e., when only the transport depends on the flow, as mostly considered in reactive transport [35].

Whereas the surfactant transport is described by a reaction-diffusion-convection equation, water flow in variably saturated porous media is modelled by the Richards 
equation $[7,18]$. The main assumption in this case is that the air remains in contact with the atmosphere, having a constant pressure (the atmospheric pressure, here assumed zero). This allows reducing the flow model to one equation, the Richards equation. In mathematical terms, this equation is degenerate parabolic, whose solution has typically low regularity [3].

From the above, and adopting the pressure head as the main unknown in the Richards equation, we study here different linearization schemes for the model

$$
\frac{\partial \theta(\Psi, c)}{\partial t}-\nabla \cdot(K(\theta(\Psi, c)) \nabla(\Psi+z))=H_{1}
$$

and

$\frac{\partial \theta(\Psi, c) c}{\partial t}-\nabla \cdot\left(D \nabla c-\mathbf{u}_{\mathbf{w}} c\right)+R(c)=H_{2}$,

holding for $\mathbf{x} \in \Omega$ ( $z$ being the vertical coordinate of $\mathbf{x}$, pointing against gravity) and $t \in(0, T]$. Here $\Omega$ is a bounded, open domain in $\mathbb{R}^{d}(d=1,2$ or 3 ) having a Lipschitz continuous boundary $\partial \Omega$ and $T>0$ is the final time. Further, $\theta(\cdot, \cdot)$ denotes the water content, and is a given function depending on the pressure head $\Psi$ and on the surfactant concentration $c$. Also, $K(\cdot)$ is the hydraulic conductivity, $D>0$ the diffusion/dispersion coefficient. Finally, $\mathbf{u}_{\mathbf{w}}:=-K(\theta, c) \nabla(\Psi+z)$ is the water flux, $R(\cdot)$ the reaction term, expressed as a function of the concentration $c$, and $H_{1}, H_{2}$ are the external sinks/sources. Initial and boundary conditions, which are specified below, complete the system.

We point out that the water content and the hydraulic conductivity, $\theta(\cdot, \cdot)$ and $K(\cdot)$ are given nonlinear functions. They are medium- and surfactant-dependent and are determined experimentally (see [18]). Specific choices are provided in Section 2.

To solve numerically the system (1)-(2) one needs to discretize in time and space. We refer to [15] for a practical review of numerical methods for the Richards equation. Due to the low regularity of the solution and the need of relatively large time steps, the backward Euler method is the best candidate for the time discretization. Multiple spatial discretization techniques are available, such as the Galerkin finite element method (FEM) [5, 32, 39], the mixed finite element method (MFEM) [4, 36, 44, 47], the multi-point flux approximation (MPFA) [1, 6, 24], and the finite volume method (FVM) $[9,13,14]$.

Since the time discretization is not explicit, the outcome is a sequence of nonlinear problems, for which a linearization step has to be performed. Widely used linearization schemes are the quadratic, locally convergent Newton method and the modified Picard method [10]. For both, the convergence is guaranteed if the starting point is close to the solution. Since for evolution equations the initial guess is typically the solution at the previous time, this may induces severe restrictions on the time step size (see [37]). There exist several modifications of the Newton scheme improving this aspect, including like line-search and trust-region methods, or Anderson acceleration techniques, as discussed, e.g., in $[15,21,26,28,45,46,49]$, or exploiting the structure of the nonlinearity appearing in the hyperbolic two-phase flow model, as discussed in [22]. Among alternative approaches we mention the L-scheme (see [30, 34, 40, 48]) and the modified L-scheme [31], both being robust w.r.t. the mesh size, but converging linearly. In particular, the L-scheme converges for any starting point, and the restriction on the time step, if any, is very mild. The modified L-scheme makes explicit use of the choice of the starting point as the solution obtained at the previous time, and has an improved convergence behavior if the changes in the solutions at two successive times are controlled by the time step. Nevertheless, the modified L-scheme involves computation of derivatives while the L-scheme does not. Finally, the robustness of the Newton method is significantly increased if one considers combinations of the Picard and the Newton methods [8], and in particular of the L-scheme and the Newton scheme [30].

We conclude this discussion by mentioning that in this paper we adopt the FEM and the FVM, but the iterative schemes presented here can be applied in combination with any other spatial discretization. The focus is on effectively solving the flow and transport system (1)-(2), and in particular on the adequate treating of the coupling between the two model components (the flow and the reactive transport). The schemes are divided into three main categories: monolithic (Mon), nonlinear splitting (NonLinS) and alternate splitting (AltS). Subsequently, we denote, e.g., by Mon-Newton, the monolithic scheme obtained by applying the Newton method as linearization. The nonlinear splitting schemes (NonLinS) should be understood as solving at each time step first the flow equation until convergence, by using the surfactant concentration from the last iteration, and then with the obtained flow solving the transport equation until convergence. The procedure can be continued iteratively, this being the usual or classical splitting method for transport problems. The convergence of NonLinS does not depend on the linearization approach used for each model component (Newton, Picard, or L-scheme), because we assume that the nonlinear subproblems are solved exactly, i.e., until convergence. Finally, the alternate splitting methods (AltS) have a different philosophy. Instead of solving each subproblem until convergence within each iteration, one performs only one step of the chosen linearization. For example, AltS-NE will perform one Newton step for each model component, and iterate. These schemes are illustrated in Figs. 1 and 2. 


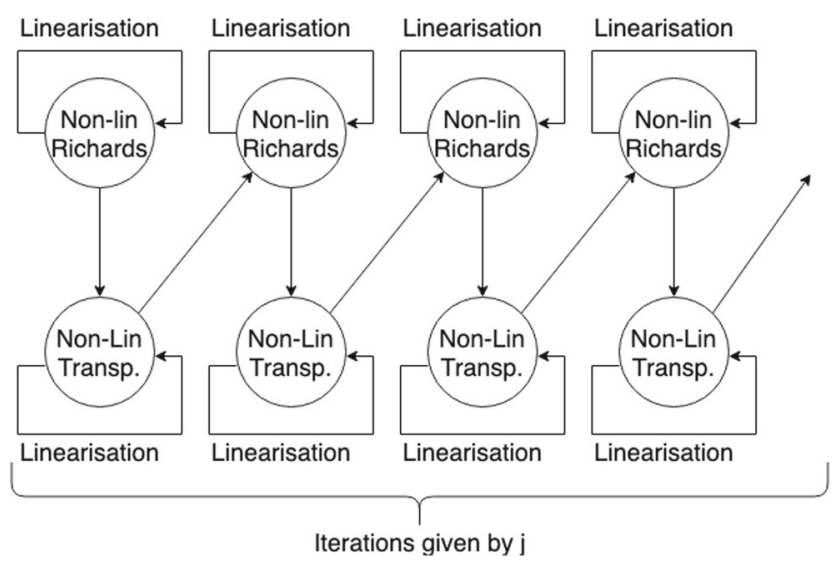

Fig. 1 The nonlinear splitting approach

All the schemes can be analyzed theoretically, and we do this exemplary for Mon-LS, i.e., for the monolithic approach combined with the L-scheme. Based on comparative numerical tests performed for academic and benchmark problems, we see that the alternate methods can save substantial computational time, while maintaining the robustness of the L-scheme.

The remaining of the paper is organized as follows. In Section 2, we establish the mathematical model and the notation used and present the iterative monolithic and splitting schemes. In Section 3, we prove the convergence of the Mon-LS scheme and briefly discuss the convergence of the other schemes. Section 4 presents five different numerical examples. They are inspired by the cases already studied in the literature $[25,30]$. Section 5 concludes this work.

\section{Problem formulation, discretization, and iterative schemes}

We solve the fully coupled system (1)-(2), completed by homogeneous Dirichlet boundary conditions for both $\Psi$ and $c$ and the initial conditions:

$\Psi=\Psi_{0}$ and $c=c_{0}$ at $t=0$.

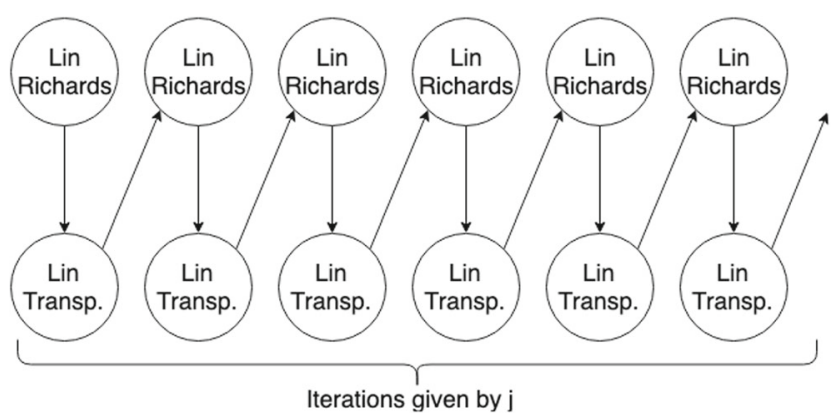

Fig. 2 The alternate splitting approach
We use the van Genuchten-Mualem parameterization [17]

$$
\begin{aligned}
& \theta(\Psi)= \begin{cases}\theta_{r}+\left(\theta_{s}-\theta_{r}\right)\left(\frac{1}{1+(-\alpha \Psi)^{n}}\right)^{\frac{n-1}{n}}, & \Psi \leq 0 \\
\theta_{S}, & \Psi>0,\end{cases} \\
& K(\theta(\Psi))= \begin{cases}K_{S} \theta_{e}(\Psi)^{\frac{1}{2}}\left[1-\left(1-\theta_{e}(\Psi)^{\frac{n}{n-1}}\right)^{\frac{n-1}{n}}\right], & \Psi \leq 0 \\
K_{S}, & \Psi>0,\end{cases}
\end{aligned}
$$

where $\theta_{r}$ and $\theta_{s}$ represent the values of the residual and saturated water content, $\theta_{e}=\left(\theta-\theta_{r}\right) /\left(\theta_{s}-\theta_{r}\right)$ is the effective water content, $K_{s}$ is the conductivity, and $\alpha$ and $n$ are model parameters depending on the soil.

Observe that in the expression above for $\theta$, the influence of the surfactant on the water flow is neglected. As reported in [20, 25, 42], the surface tension between water and air does depend on the surfactant concentration $c$, implying the same for the function $\theta$ above. The following parametrization is proposed in [25]

$\theta(\Psi, c):=\theta(\gamma(c) \Psi), \quad$ with $\quad \gamma(c)=\frac{1}{1-b \log (c / a+1)}$.

Here $\theta()$ is given in (3) and $\gamma()$ is the surface tensions as depending on the concentration $c$. The parameters $a$ and $b$ depend on the fluid and the medium. We refer to [41, 42] for details about (5).

This gives the following expressions for $\theta$ and $K$

$\theta(\Psi, c)= \begin{cases}\theta_{r}+\left(\theta_{s}-\theta_{r}\right)\left[1 /\left(1+\left(-\alpha\left(\frac{1}{1-b \log (c / a+1)}\right) \Psi\right)^{\eta}\right)\right]^{\frac{n-1}{n}}, & \Psi \leq 0 \\ \theta_{s}, & \Psi>0\end{cases}$

$K(\theta(\Psi, c))= \begin{cases}K_{S} \theta_{e}(\Psi, c)^{\frac{1}{2}}\left[1-\left(1-\theta_{e}(\Psi, c)^{\frac{n}{n-1}}\right)^{\frac{n-1}{n}}\right], & \Psi \leq 0 \\ K_{S}, & \Psi>0 .\end{cases}$

This shows that the flow component also depends on the reactive transport, implying that the model is coupled in both directions.

In the following, we proceed by discretizing (1) and (2) in time and space. We will use common notations in functional analysis. We denote by $L^{2}(\Omega)$ the space of real valued, squared integrable functions defined on $\Omega$ and $H^{1}(\Omega)$ its subspace, containing the functions having also the first order derivatives in $L^{2}(\Omega) . H_{0}^{1}(\Omega)$ is the space of functions belonging to $H^{1}(\Omega)$ and vanishing on $\partial \Omega$. Further, we denote by $<\cdot, \cdot>$ the $L^{2}(\Omega)$ scalar product (and by $\|\cdot\|$ the associated norm) or the pairing between $H_{0}^{1}$ and its dual $H^{-1}$. Finally, by $L^{2}(0, T ; X)$, we mean the Bochner 
space of functions taking values in the Banach-space $X$, the extension to $H^{1}(0, T ; X)$ being straightforward.

With this, we state the weak formulation of the problem related to (1)-(2):

Problem P: Find $\Psi, c \quad \in \quad L^{2}\left(0, T ; H_{0}^{1}(\Omega)\right) \quad \cap$ $H^{1}\left(0, T ; H^{-1}(\Omega)\right)$ such that

$$
\begin{aligned}
& <\partial_{t} \theta(\Psi, c), v_{1}>+<K(\theta(\Psi, c)) \nabla(\Psi+z), \nabla v_{1}> \\
= & <H_{1}, v_{1}>
\end{aligned}
$$

and

$<\partial_{t}(\theta(\Psi, c) c), v_{2}>+<D \nabla c+\mathbf{u}_{\mathbf{w}} c, \nabla v_{2}>=<H_{2}, v_{2}>$

hold for all $v_{1}, v_{2} \in H_{0}^{1}(\Omega)$ and almost every $t \in(0, T]$.

We now combine the backward Euler method with linear Galerkin finite elements for the discretization of Problem P. We let $N \in \mathbb{N}$ be a strictly positive natural number and the time step $\tau:=T / N$. Correspondingly, the discrete times are $t_{n}:=n \tau(n \in\{0,1, \ldots, N\})$. Further, we let $T_{h}$ be a regular decomposition of $\Omega, \bar{\Omega}=\underset{T \in T_{h}}{\cup} T$ into $d$ dimensional simplices, with $h$ denoting the mesh diameter. The finite element space $V_{h} \subset H_{0}^{1}(\Omega)$ is defined by

$V_{h}:=\left\{v_{h} \in H_{0}^{1}(\Omega)\right.$ s.t. $v_{h \mid T} \in \mathbb{P}_{1}(T)$, for any $\left.T \in T_{h}\right\}$,

where $\mathbb{P}_{1}(T)$ denotes the space of linear polynomials on $T$ and $v_{h \mid T}$ the restriction of $v_{h}$ to $T$.

For the fully discrete counterpart of Problem P, we let $n \geq 1$ be fixed and assume that $\Psi_{h}^{n-1}, c_{h}^{n-1} \in V_{h}$ are given. The solution pair at time $t_{n}$ solves

Problem $\mathbf{P}_{n}$ : Find $\Psi_{h}^{n}, c_{h}^{n} \in V_{h}$ such that for all $v_{h}, w_{h} \in$ $V_{h}$ there holds

$$
\begin{aligned}
< & \theta\left(\Psi_{h}^{n}, c_{h}^{n}\right)-\theta\left(\Psi_{h}^{n-1}, c_{h}^{n-1}\right), v_{h}> \\
& +\tau<K\left(\theta\left(\Psi_{h}^{n}, c_{h}^{n}\right)\right)\left(\nabla\left(\Psi_{h}^{n}\right)+\mathbf{e}_{\mathbf{z}}\right), \nabla v_{h}> \\
= & \tau<H_{1}, v_{h}>
\end{aligned}
$$

and

$$
\begin{aligned}
< & \theta\left(\Psi_{h}^{n}, c_{h}^{n}\right) c_{h}^{n}-\theta\left(\Psi_{h}^{n-1}, c_{h}^{n-1}\right) c_{h}^{n-1}, w_{h}> \\
& +\tau<D \nabla c_{h}^{n}+\mathbf{u}_{\mathbf{w}}^{\mathbf{n}-1} c_{h}^{n}, \nabla w_{h}>=\tau<H_{2}, w_{h}>.
\end{aligned}
$$

$\mathbf{e}_{\mathbf{z}}$ denotes the unit vector in the direction opposite to gravity.

Remark 1 Observe that $\mathbf{u}_{\mathbf{w}}^{\mathbf{n}-1}$ appears in the convective term in (12). This choice is made for the ease of presentation. Nevertheless, all calculations carried out in this paper were doubled by ones where $\mathbf{u}_{\mathbf{w}}^{\mathbf{n}}$ has replaced $\mathbf{u}_{\mathbf{w}}^{\mathbf{n}-\mathbf{1}}$. The differences in the results were marginal.
Observe that Problem $\mathrm{P}_{n}$ is a coupling system of two elliptic, nonlinear equations. In the following, we discuss different iterative schemes for solving this system.

\subsection{Iterative linearization schemes}

We discuss monolithic and splitting approaches for solving Problem $\mathrm{P}_{n}$, combined with either the Newton method, the modified Picard [10], or the L-scheme [30, 34]. In the following the index $n$ always refers to the time step, whereas $j$ denotes the iteration index. As a rule, the iterations start with the solution at the previous time, $t_{n-1}$.

In the monolithic approach, one solves the two equations of the system (11)-(12) at once, combined with a linearization method. Formally, this becomes

Problem PMon PM $_{n+1}$ : Find $\Psi^{n, j+1}$ and $c^{n, j+1}$ such that

$$
\left\{\begin{array}{l}
F_{1}^{l i n}\left(\Psi^{n, j+1}, c^{n, j+1}\right)=0, \\
F_{2}^{l i n}\left(\Psi^{n, j+1}, c^{n, j+1}\right)=0 .
\end{array}\right.
$$

$F_{k}^{L i n}$ is a linearization of the expression $F_{k}(k=1,2)$ appearing in the system (11)-(12). Depending on the used linearization technique, one speaks about a monolithic Newton scheme (Mon-Newton), or monolithic Picard (Mon-Picard) or monolithic L-scheme (Mon-LS). These three schemes will be presented in detail below.

In the iterative splitting approach one solves each equation separately and then iterates between these, using the results previously obtained. We distinguish between two main splitting ways: the nonlinear splitting and the alternate splitting. These are schematized in Figs. 1 and 2 respectively. The former becomes :

Problem PNonLinS $S_{n, j+1}: \quad$ Find $\Psi^{n, j+1}$ and $c^{n, j+1}$ such that

$$
\left\{\begin{array}{c}
F_{1}\left(\Psi^{n, j+1}, c^{n, j}\right)=0, \text { followed by } \\
F_{2}\left(\Psi^{n, j+1}, c^{n, j+1}\right)=0 .
\end{array}\right.
$$

For the linearization of $F_{1}$ and $F_{2}$, one can use one of the three linearization techniques mentioned before. In contrast, in the alternate splitting, one performs only one linearization step per iteration (see also Fig. 2). The alternate splitting scheme becomes

Problem PAltS P $_{n, j+1}: \quad$ Find $\Psi^{n, j+1}$ and $c^{n, j+1}$ such that

$$
\left\{\begin{aligned}
F_{1}^{l i n}\left(\Psi^{n, j+1}, c^{n, j}\right) & =0, \text { followed by } \\
F_{2}^{l i n}\left(\Psi^{n, j+1}, c^{n, j+1}\right) & =0
\end{aligned}\right.
$$

Depending on which linearization is used, one speaks about alternate splitting Newton (AltS-NE), alternate splitting Picard (AltS-Picard), or alternate splitting L-scheme (AltSLS). The schemes are presented in detail below. 


\subsubsection{The monolithic Newton method (Mon-Newton)}

We recall that the Newton scheme is quadratically, but only locally convergent. The monolithic Newton method applied to (11)-(12) gives

Problem PMon-Newton ${ }_{n, j+1}$ : Let $\Psi_{h}^{n-1}, c^{n-1}, \Psi_{h}^{n, j}, c_{h}^{n, j}$ $\in V_{h}$ be given, find $\Psi_{h}^{n, j+1}, c_{h}^{n, j+1} \in V_{h}$ such that for all $v_{h}, w_{h} \in V_{h}$ one has

$$
\begin{aligned}
< & \theta\left(\Psi_{h}^{n, j}, c_{h}^{n, j}\right)-\theta\left(\Psi_{h}^{n-1}, c_{h}^{n-1}\right), v_{h}> \\
& +<\frac{\partial \theta}{\partial \Psi}\left(\Psi_{h}^{n, j}, c_{h}^{n, j}\right)\left(\Psi_{h}^{n, j+1}-\Psi_{h}^{n, j}\right), v_{h}> \\
& +\tau<K\left(\theta\left(\Psi_{h}^{n, j}, c_{h}^{n, j}\right)\right)\left(\nabla \Psi_{h}^{n, j+1}+\mathbf{e}_{\mathbf{z}}\right), \nabla v_{h}> \\
& +\tau<K^{\prime}\left(\theta\left(\Psi_{h}^{n, j}, c_{h}^{n, j}\right)\right) \frac{\partial \theta}{\partial \Psi}\left(\Psi_{h}^{n, j}, c_{h}^{n, j}\right) \\
& \times\left(\nabla \Psi_{h}^{n, j}+\mathbf{e}_{\mathbf{z}}\right)\left(\Psi_{h}^{n, j+1}-\Psi_{h}^{n, j}\right), \nabla v_{h}> \\
= & \tau<H_{1}, v_{h}>
\end{aligned}
$$

and

$$
\begin{aligned}
< & \theta\left(\Psi_{h}^{n, j}, c_{h}^{n, j}\right) c_{h}^{n, j+1}-\theta\left(\Psi_{h}^{n-1}, c_{h}^{n-1}\right) c_{h}^{n-1}, w_{h}> \\
& +<\frac{\partial \theta}{\partial c}\left(\Psi_{h}^{n, j}, c_{h}^{n, j}\right)\left(c_{h}^{n, j+1}-c_{h}^{n, j}\right), v_{h}> \\
& +\tau<D \nabla c_{h}^{n, j+1}+\mathbf{u}_{\mathbf{w}}^{\mathbf{n}-1} c_{h}^{n, j+1}, \nabla w_{h}> \\
= & \tau<H_{2}, w_{h}>.
\end{aligned}
$$

For the ease of presentation, here a simplified monolithic formulation is given, involving only the derivative of $\theta$ with respect to $\Psi$ in (16), and only the derivative of $\theta$ with respect to $c$ in (17). In the full monolithic approach, both partial derivatives should be involved for all nonlinear functions, e.g.,

$$
\begin{aligned}
& \theta\left(\Psi_{h}^{n, j+1}, c_{h}^{n, j+1}\right) \rightarrow \theta\left(\Psi_{h}^{n, j}, c_{h}^{n, j}\right) \\
& +\left(\frac{\partial \theta}{\partial \Psi}\right)\left(\Psi_{h}^{n, j}, c_{h}^{n, j}\right)\left(\Psi_{h}^{n, j+1}-\Psi_{h}^{n, j}\right) \\
& +\left(\frac{\partial \theta}{\partial c}\right)\left(\Psi_{h}^{n, j}, c_{h}^{n, j}\right)\left(c_{h}^{n, j+1}-c_{h}^{n, j}\right) .
\end{aligned}
$$

However, we have carried out computations with the full monolithic approach and the results were practically showing no difference.

\subsubsection{The monolithic Picard method (Mon-Picard)}

The modified Picard method was initially proposed by Celia [10] for the Richards equation. It is similar to the Newton method in dealing with the nonlinearity in the saturation, but not in the permeability. Being a modification of the Newton method, the modified Picard method is only linearly convergent [37]. The monolithic Picard method applied to (11)-(12) becomes
Problem PMon-Picard ${ }_{n, j+1}$ : Let $\Psi_{h}^{n-1}, c_{h}^{n-1}, \Psi_{h}^{n, j}, c_{h}^{n, j}$ $\in V_{h}$ be given, find $\Psi_{h}^{n, j+1}, c_{h}^{n, j+1} \in V_{h}$ such that for all $v_{h}, w_{h} \in V_{h}$, one has

$$
\begin{aligned}
& <\theta\left(\Psi_{h}^{n, j}, c_{h}^{n, j}\right)-\theta\left(\Psi_{h}^{n-1}, c_{h}^{n-1}\right), v_{h}> \\
& \quad+<\frac{\partial \theta}{\partial \Psi}\left(\Psi_{h}^{n, j}, c_{h}^{n, j}\right)\left(\Psi_{h}^{n, j+1}-\Psi_{h}^{n, j}\right), v_{h}> \\
& \quad+\tau<K\left(\theta\left(\Psi_{h}^{n, j}, c_{h}^{n, j}\right)\right)\left(\nabla \Psi_{h}^{n, j+1}+\mathbf{e}_{\mathbf{z}}\right), \nabla v_{h}> \\
& =\tau<H_{1}, v_{h}>
\end{aligned}
$$

and

$$
\begin{aligned}
< & \theta\left(\Psi_{h}^{n, j}, c_{h}^{n, j}\right) c_{h}^{n, j+1}-\theta\left(\Psi_{h}^{n-1}, c_{h}^{n-1}\right) c_{h}^{n-1}, w_{h}> \\
& +<\frac{\partial \theta}{\partial c}\left(\Psi_{h}^{n, j}, c_{h}^{n, j}\right)\left(c_{h}^{n, j+1}-c_{h}^{n, j}\right), w_{h}> \\
& +\tau<D \nabla c_{h}^{n, j+1}+\mathbf{u}_{\mathbf{w}}^{\mathbf{n}-1} c_{h}^{n, j+1}, \nabla w_{h}> \\
= & \tau<H_{2}, w_{h}>.
\end{aligned}
$$

As before, a Picard iteration for the full monolithic approach would involve both partial derivatives of $\theta$.

\subsubsection{The monolithic L-scheme (Mon-LS)}

The monolithic L-scheme for solving (11)-(12) becomes

Problem PMon-LS $\mathbf{S}_{n, j+1}$ : Let $\Psi_{h}^{n-1}, \Psi_{h}^{n, j}, c_{h}^{n-1}, c_{h}^{n, j} \in$ $V_{h}$ be given and with $L_{1}, L_{2}>0$ large enough (as specified below), find $\Psi_{h}^{n, j+1}, c_{h}^{n, j+1} \in V_{h}$ s.t. for all $v_{h}, w_{h} \in V_{h}$

$$
\begin{aligned}
< & \theta\left(\Psi_{h}^{n, j}, c_{h}^{n, j}\right)-\theta\left(\Psi_{h}^{n-1}, c_{h}^{n-1}\right), v_{h}> \\
& +L_{1}<\Psi_{h}^{n, j+1}-\Psi_{h}^{n, j}, v_{h}> \\
& +\tau<K\left(\theta\left(\Psi_{h}^{n, j}, c_{h}^{n, j}\right)\right)\left(\nabla \Psi_{h}^{n, j+1}+\mathbf{e}_{\mathbf{z}}\right), \nabla v_{h}> \\
= & \tau<H_{1}, v_{h}>, \\
< & \theta\left(\Psi_{h}^{n, j}, c_{h}^{n, j}\right) c_{h}^{n, j+1}-\theta\left(\Psi_{h}^{n-1}, c_{h}^{n-1}\right) c_{h}^{n-1}, w_{h}> \\
& +L_{2}<c_{h}^{n, j+1}-c_{h}^{n, j}, w_{h}> \\
& +\tau<D \nabla c_{h}^{n, j+1}+\mathbf{u}_{\mathbf{w}}^{\mathbf{n}-1} c_{h}^{n, j+1}, \nabla w_{h}> \\
= & \tau<H_{2}, w_{h}>.
\end{aligned}
$$

The parameters $L_{1}$ and $L_{2}$ should be large enough to ensure the convergence of the scheme (see Section 3 ). In practice, the values of $L_{1}$ and $L_{2}$ are connected to the maximal values of $\partial_{\Psi} \theta$ and $\partial_{c} \theta$ (recall that $\theta$ is assumed increasing in $\Psi$ and in $c$ ).

The L-scheme does not involve the computations of derivatives, and the linear systems to be solved within each iteration are better conditioned compared with the ones 
given by Newton or Picard methods (see [30]). Moreover, this scheme is (linearly) convergent for any initial guess for the iteration. Finally, the classical full monolithic approach is obtained by involving $L_{1}$ and $L_{2}$ in both of the equations.

\subsubsection{The nonlinear splitting approach (NonLinS)}

The nonlinear splitting approach for solving (11)-(12) becomes

Problem PNonLinS $S_{n, j+1}$ : Let $\Psi_{h}^{n-1}, c^{n-1}, \Psi_{h}^{n, j}, c_{h}^{n, j} \in$ $V_{h}$ be given, find $\Psi_{h}^{n, j+1} \in V_{h}$ s.t.

$$
\begin{aligned}
< & \theta\left(\Psi_{h}^{n, j+1}, c_{h}^{n, j}\right)-\theta\left(\Psi_{h}^{n-1}, c_{h}^{n-1}\right), v_{h}> \\
& +\tau<K\left(\theta\left(\Psi_{h}^{n, j}, c_{h}^{n, j}\right)\right)\left(\nabla \Psi_{h}^{n, j+1}+\mathbf{e}_{\mathbf{z}}\right), \nabla v_{h}> \\
= & \tau<H_{1}, v_{h}>
\end{aligned}
$$

holds true for all $v_{h} \in V_{h}$. Then, with $\Psi_{h}^{n, j+1}$ obtained, find $c_{h}^{n, j+1} \in V_{h}$ such that for all $w_{h} \in V_{h}$, it holds

$$
\begin{aligned}
< & \theta\left(\Psi_{h}^{n, j+1}, c_{h}^{n, j+1}\right) c_{h}^{n, j+1}-\theta\left(\Psi_{h}^{n-1}, c_{h}^{n-1}\right) c_{h}^{n-1}, w_{h}> \\
& +\tau<D \nabla c_{h}^{n, j+1}+\mathbf{u}_{\mathbf{w}}^{\mathbf{n}-1} c_{h}^{n, j+1}, \nabla w_{h}> \\
= & \tau<H_{2}, w_{h}>.
\end{aligned}
$$

As for the monolithic schemes, one can apply the different linear iterative schemes to obtain fully linear versions of the splitting approach. This is done first to solve (23) and, once a solution to (23) is available, this is employed in the linearization of (24).

\subsubsection{The alternate Newton method (AltS-Newton)}

In the alternate Newton method applied to (11)-(12), one solves

Problem PAItS-Newton $_{n, j+1}$ : Let $\Psi_{h}^{n-1}, c^{n-1}, \Psi_{h}^{n, j}, c_{h}^{n, j}$ $\in V_{h}$ be given, find $\Psi_{h}^{n, j+1} \in V_{h}$ s.t.

$$
\begin{aligned}
< & \theta\left(\Psi_{h}^{n, j}, c_{h}^{n, j}\right)-\theta\left(\Psi_{h}^{n-1}, c_{h}^{n-1}\right), v_{h}> \\
& +<\frac{\partial \theta}{\partial \Psi}\left(\Psi_{h}^{n, j}, c_{h}^{n, j}\right)\left(\Psi_{h}^{n, j+1}-\Psi_{h}^{n, j}\right), v_{h}> \\
& +\tau<K\left(\theta\left(\Psi_{h}^{n, j}, c_{h}^{n, j}\right)\right)\left(\nabla \Psi_{h}^{n, j+1}+\mathbf{e}_{\mathbf{z}}\right), \nabla v_{h}> \\
& +\tau<K^{\prime}\left(\theta\left(\Psi_{h}^{n, j}, c_{h}^{n, j}\right)\right) \frac{\partial \theta}{\partial \Psi}\left(\Psi_{h}^{n, j}, c_{h}^{n, j}\right) \\
& \times\left(\nabla \Psi_{h}^{n, j}+\mathbf{e}_{\mathbf{z}}\right)\left(\Psi_{h}^{n, j+1}-\Psi_{h}^{n, j}\right), \nabla v_{h}> \\
= & \tau<H_{1}, v_{h}>
\end{aligned}
$$

holds true for all $v_{h} \in V_{h}$. Then, with $\Psi_{h}^{n, j+1}$ obtained above, find $c_{h}^{n, j+1} \in V_{h}$ such that for all $w_{h} \in V_{h}$, one has

$$
\begin{aligned}
< & \theta\left(\Psi_{h}^{n, j+1}, c_{h}^{n, j}\right) c_{h}^{n, j+1}-\theta\left(\Psi_{h}^{n-1}, c_{h}^{n-1}\right) c_{h}^{n-1}, w_{h}> \\
& +<\frac{\partial \theta}{\partial c}\left(\Psi_{h}^{n, j+1}, c_{h}^{n, j}\right)\left(c_{h}^{n, j+1}-c_{h}^{n, j}\right), v_{h}> \\
& +\tau<D \nabla c_{h}^{n, j+1}+\mathbf{u}_{\mathbf{w}}^{\mathbf{n}-1} c_{h}^{n, j+1}, \nabla w_{h}> \\
= & \tau<H_{2}, w_{h}>.
\end{aligned}
$$

\subsubsection{The alternate Picard method (AltS-Picard)}

The alternate Picard method applied to (11)-(12) becomes

Problem PAltS-Picard ${ }_{n, j+1}$ : Let $\Psi_{h}^{n-1}, c^{n-1}, \Psi_{h}^{n, j}, c_{h}^{n, j}$ $\in V_{h}$ be given, find $\Psi_{h}^{n, j+1} \in V_{h}$ s.t.

$$
\begin{aligned}
& <\theta\left(\Psi_{h}^{n, j}, c_{h}^{n, j}\right)-\theta\left(\Psi_{h}^{n-1}, c_{h}^{n-1}\right), v_{h}> \\
& \quad+<\frac{\partial \theta}{\partial \Psi}\left(\Psi_{h}^{n, j}, c_{h}^{n, j}\right)\left(\Psi_{h}^{n, j+1}-\Psi_{h}^{n, j}\right), v_{h}> \\
& \quad+\tau<K\left(\theta\left(\Psi_{h}^{n, j}, c_{h}^{n, j}\right)\right)\left(\nabla\left(\Psi_{h}^{n, j+1}\right)+\mathbf{e}_{\mathbf{z}}\right), \nabla v_{h}> \\
& =\tau<H_{1}, v_{h}>
\end{aligned}
$$

hold true for all $v_{h} \in V_{h}$. Then, with $\Psi_{h}^{n, j+1}$ obtained above, find $c_{h}^{n, j+1} \in V_{h}$ such that for all $w_{h} \in V_{h}$, one has

$$
\begin{aligned}
< & \theta\left(\Psi_{h}^{n, j+1}, c_{h}^{n, j}\right) c_{h}^{n, j+1}-\theta\left(\Psi_{h}^{n-1}, c_{h}^{n-1}\right) c_{h}^{n-1}, w_{h}> \\
& +<\frac{\partial \theta}{\partial c}\left(\Psi_{h}^{n, j+1}, c_{h}^{n, j}\right)\left(c_{h}^{n, j+1}-c_{h}^{n, j}\right), w_{h}> \\
& +\tau<D \nabla c_{h}^{n, j+1}+\mathbf{u}_{\mathbf{w}}^{\mathbf{n}-1} c_{h}^{n, j+1}, \nabla w_{h}> \\
= & \tau<H_{2}, w_{h}>.
\end{aligned}
$$

\subsubsection{The alternate L-scheme (AltS-LS)}

The alternate L-scheme for solving (11)-(12) becomes

Problem PAItS-LS $\mathbf{L}_{n, j+1}$ : Let $\Psi_{h}^{n-1}, c^{n-1}, \Psi_{h}^{n, j}, c_{h}^{n, j} \in$ $V_{h}$ be given, find $\Psi_{h}^{n, j+1} \in V_{h}$ s.t.

$$
\begin{aligned}
< & \theta\left(\Psi_{h}^{n, j}, c_{h}^{n, j}\right)-\theta\left(\Psi_{h}^{n-1}, c_{h}^{n-1}\right), v_{h}> \\
& +L_{1}<\Psi_{h}^{n, j+1}-\Psi_{h}^{n, j}, v_{h}> \\
& +\tau<K\left(\theta\left(\Psi_{h}^{n, j}, c_{h}^{n, j}\right)\right)\left(\nabla \Psi_{h}^{n, j+1}+\mathbf{e}_{\mathbf{z}}\right), \nabla v_{h}> \\
= & \tau<H_{1}, v_{h}>
\end{aligned}
$$

hold true for all $v_{h} \in V_{h}$. Then, with $\Psi_{h}^{n, j+1}$ obtained above, find $c_{h}^{n, j+1} \in V_{h}$ such that for all $w_{h} \in V_{h}$, one has

$$
\begin{aligned}
< & \theta\left(\Psi_{h}^{n, j+1}, c_{h}^{n, j}\right) c_{h}^{n, j+1}-\theta\left(\Psi_{h}^{n-1}, c_{h}^{n-1}\right) c_{h}^{n-1}, w_{h}> \\
& +L_{2}<c_{h}^{n, j+1}-c_{h}^{n, j}, w_{h}> \\
& +\tau<D \nabla c+\mathbf{u}_{\mathbf{w}}^{\mathbf{n}-1} c_{h}^{n, j+1}, \nabla w_{h}>=\tau<H_{2}, w_{h}>.
\end{aligned}
$$


Remark 2 (Stopping criterion) For all schemes (monolithic or splitting), the iteration is stopped if for some small numbers $\epsilon_{1}, \epsilon_{2}>0$ one has

$\left\|\Psi_{h}^{n, j+1}-\Psi_{h}^{n, j}\right\| \leq \epsilon_{1}$, and $\left\|c_{h}^{n, j+1}-c_{h}^{n, j}\right\| \leq \epsilon_{2}$,

later in the numerical section we will consider $\epsilon_{1}=\epsilon_{2}$.

\section{Convergence analysis}

In this section, we analyze the convergence of the monolithic L-scheme introduced through Problem PMon$\mathrm{LS}_{n, j+1}$. We restrict the analysis to this iteration, but mention that the convergence analysis for the other (monolithic and splitting) schemes introduced above can be done in a similar fashion. We start by defining the errors

$e_{\Psi}^{j+1}:=\Psi_{h}^{n, j+1}-\Psi_{h}^{n, j}$ and $e_{c}^{j+1}:=c_{h}^{n, j+1}-c_{h}^{n, j}$,

obtained at iteration $j+1$. The scheme is convergent if both errors vanish when $j \rightarrow \infty$.

The convergence is obtained under the following assumptions:

(A1) There exist $\alpha_{\Psi}>0$ and $\alpha_{c} \geq 0$ such that for any $\Psi_{1}, \Psi_{2} \in \mathbb{R}$ and $c_{1}, c_{2} \in \mathbb{R}_{+}$

$$
\begin{aligned}
& <\theta\left(\Psi_{1}, c_{1}\right)-\theta\left(\Psi_{2}, c_{2}\right), \Psi_{1}-\Psi_{2}> \\
& \quad+<c_{1} \theta\left(\Psi_{1}, c_{1}\right)-c_{2} \theta\left(\Psi_{2}, c_{2}\right), c_{1}-c_{2}> \\
& \geq \alpha_{\Psi}\left\|\theta\left(\Psi_{1}, c_{1}\right)-\theta\left(\Psi_{2}, c_{2}\right)\right\|^{2}+\alpha_{c}\left\|\Psi_{1}-\Psi_{2}\right\|^{2} .
\end{aligned}
$$

Furthermore, there exist two constants $\theta_{m} \geq 0$ and $\theta_{M}<\infty$ such that $\theta_{m} \leq \theta(\Psi, c) \leq \theta_{M}, \forall \Psi, c \in \mathbb{R}$

The function $K(\theta(\cdot, \cdot))$ is Lipschitz continuous, with respect to both variables, and there exist two constants $K_{m}$ and $K_{M}$ such that $0 \leq K_{m} \leq K \leq$ $K_{M}<\infty$.

(A3) There exist also $M_{u}, M_{\Psi}, M_{c} \geq 0$ such that $\left\|\mathbf{u}_{\mathbf{w}}^{\mathbf{n}}\right\|_{L^{\infty}} \leq M_{u},\left\|\nabla \Psi^{n}\right\|_{L^{\infty}} \leq M_{\Psi}$ and $\left\|c^{n}\right\|_{L^{\infty}} \leq$ $M_{c}$ for all $n \in \mathbb{N}$.

Remark 3 (A2) is satisfied in most realistic situations. (A3) is a pure technical requirement, being satisfied when data is sufficiently regular, which is assumed to be the case for the present analysis. The inequality (32) in (A1) is a coercivity assumption. It is in particular satisfied if $\theta$ only depends on $\Psi$, and for common relationships $\theta--\Psi$ encountered in the engineering literature.

Theorem 1 Let $n \in\{1,2, \ldots N\}$ be given and assume (A1)-(A3) be satisfied. If the time step is small enough (see 42 below), the monolithic L-scheme in (29)-(30) is linearly convergent for any $L_{1}$ and $L_{2}$ satisfying (41).
Proof We follow the ideas in [30, 34] and start by subtracting (11) from (29) to obtain the error equation

$$
\begin{aligned}
< & \theta_{h}^{n, j}-\theta_{h}^{n}, v_{h}>+L_{1}<\Psi_{h}^{n, j+1}-\Psi_{h}^{n, j}, v_{h}> \\
& +\tau<K_{h}^{n, j} \nabla e_{\Psi}^{n, j+1}, \nabla v_{h}> \\
& +\tau<\left(K_{h}^{n, j}-K_{h}^{n}\right) \nabla \Psi_{h}^{n, j+1}, \nabla v_{h}> \\
& +\tau<\left(K_{h}^{n, j}-K_{h}^{n}\right) \mathbf{e}_{\mathbf{z}}, \nabla v_{h}>=0,
\end{aligned}
$$

where $\theta_{h}^{n, j}:=\theta\left(\Psi_{h}^{n, j}, c_{h}^{n, j}\right), \theta_{h}^{n-1}:=\theta\left(\Psi_{h}^{n}, c_{h}^{n}\right), K_{h}^{n, j}:=$ $K_{h}\left(\theta_{h}^{n, j}\right)$ and $K_{h}^{n}:=K_{h}\left(\theta_{h}^{n}\right)$. Testing now the above equation with $v_{h}=e_{\Psi}^{j+1}$, one obtains

$$
\begin{aligned}
< & \theta_{h}^{n, j}-\theta_{h}^{n}, e_{\Psi}^{j+1}>+L_{1} \\
& +\tau<K^{n, j} \nabla e_{\Psi}^{n, j+1}, \nabla e_{\Psi}^{j+1}> \\
& +\tau<\left(K_{h}^{n, j}-K_{h}^{n}\right) \nabla \Psi_{h}^{n, j+1}, \nabla e_{\Psi}^{j+1}> \\
& +\tau<\left(K_{h}^{n, j}-K_{h}^{n}\right) \mathbf{e}_{\mathbf{z}}, \nabla e_{\Psi}^{j+1}>=0 .
\end{aligned}
$$

By (A2) and after some algebraic manipulations, we further get

$$
\begin{aligned}
< & \theta_{h}^{n, j}-\theta_{h}^{n}, e_{\Psi}^{j}>+\frac{L_{1}}{2}\left\|e_{\Psi}^{j+1}\right\|^{2}+\frac{L_{1}}{2}\left\|e_{\Psi}^{j+1}-e_{\Psi}^{j}\right\|^{2} \\
& +\tau K_{m}\left\|\nabla e_{\Psi}^{j+1}\right\|^{2} \\
\leq & \frac{L_{1}}{2}\left\|e_{\Psi}^{j}\right\|^{2}-<\theta_{h}^{n, j}-\theta_{h}^{n}, e_{\Psi}^{j+1}-e_{\Psi}^{j}> \\
& -\tau<\left(K_{h}^{n, j}-K_{h}^{n}\right) \nabla \Psi_{h}^{n, j+1}, \nabla e_{\Psi}^{j+1} \\
> & -\tau<\left(K_{h}^{n, j}-K_{h}^{n}\right) \mathbf{e}_{\mathbf{z}}, \nabla e_{\Psi}^{j+1}>.
\end{aligned}
$$

Using now (A1), (A3), the Lipschitz continuity of $K$, and twice the Young and Cauchy-Schwarz inequalities, for any $\delta_{0}>0$ and $\delta_{1}>0$, from (35), one obtains

$$
\begin{aligned}
& <\theta_{h}^{n, j}-\theta_{h}^{n}, e_{\Psi}^{j}>+\frac{L_{1}}{2}\left\|e_{\Psi}^{j+1}\right\|^{2} \\
& +\frac{L_{1}}{2}\left\|e_{\Psi}^{j+1}-e_{\Psi}^{j}\right\|^{2}+\tau K_{m}\left\|\nabla e_{\Psi}^{j+1}\right\|^{2} \\
& \leq \frac{L_{1}}{2}\left\|e_{\Psi}^{j}\right\|^{2}+\frac{\delta_{0}}{2}\left\|\theta_{h}^{n, j}-\theta_{h}^{n}\right\|^{2}+\frac{1}{2 \delta_{0}}\left\|e_{\Psi}^{j+1}-e_{\Psi}^{j}\right\|^{2} \\
& \quad+\frac{\tau\left(M_{\Psi}^{2}+1\right) L_{k}^{2}}{2 \delta_{1}}\left\|\theta_{h}^{n, j}-\theta_{h}^{n}\right\|^{2}+\tau \delta_{1}\left\|\nabla e_{\Psi}^{j+1}\right\|^{2} .
\end{aligned}
$$

Similarly, subtracting (12) from (30) and choosing $w_{h}=$ $e_{c}^{j+1}$ in the results, one gets

$$
\begin{gathered}
<c_{h}^{n, j+1} \theta_{h}^{n, j}-c_{h}^{n} \theta_{h}^{n}, e_{c}^{j+1}>+L_{2}<e_{c}^{j+1}-e_{c}^{j}, e_{c}^{j+1}> \\
+\tau<D \nabla e_{c}^{j+1}+\mathbf{u}_{\mathbf{w}}^{\mathbf{n}-1} e_{c}^{j+1}, \nabla e_{c}^{j+1}>=0 .
\end{gathered}
$$


This can be rewritten as

$$
\begin{aligned}
< & c_{h}^{n, j} \theta_{h}^{n, j}-c^{n} \theta_{h}^{n}, e_{c}^{j}>+<\theta_{h}^{n, j} e_{c}^{j+1}, e_{c}^{j+1}> \\
& +\frac{L_{2}}{2}\left\|e_{c}^{j+1}\right\|^{2}+\frac{L_{2}}{2}\left\|e_{c}^{j+1}-e_{c}^{j}\right\|^{2} \\
& +\tau D<\nabla e_{c}^{j+1}, \nabla e_{c}^{j+1}> \\
& +\frac{L_{2}}{2}\left\|e_{c}^{j}\right\|^{2}+<\theta_{h}^{n} c_{h}^{n}-\theta_{h}^{n, j} c_{h}^{n, j}, e_{c}^{j+1}-e_{c}^{j}>-\tau \\
< & \mathbf{u}_{\mathbf{w}}^{\mathbf{n}-1} e_{c}^{j+1}, \nabla e_{c}^{j+1}>.
\end{aligned}
$$

Using again (A1), (A3), and the Cauchy-Schwarz and Young inequalities, from (38), it follows that for any $\delta_{2}, \delta_{3}, \delta_{4}>0$, one has

$$
\begin{aligned}
< & c_{h}^{n, j} \theta_{h}^{n, j}-c_{h}^{n} \theta_{h}^{n}, e_{c}^{j}>+\theta_{m}\left\|e_{c}^{j+1}\right\|^{2}+\frac{L_{2}}{2}\left\|e_{c}^{j+1}\right\|^{2} \\
& +\frac{L_{2}}{2}\left\|e_{c}^{j+1}-e_{c}^{j}\right\|^{2}+\tau D\left\|\nabla e_{c}^{j+1}\right\|^{2} \\
\leq & \frac{L_{2}}{2}\left\|e_{c}^{j}\right\|^{2}+\frac{\delta_{2}}{2}\left\|\theta_{h}^{n}-\theta_{h}^{n, j}\right\|^{2}+\frac{\delta_{3}}{2}\left\|e_{c}^{j}\right\|^{2} \\
& +\left(\frac{M_{c}^{2}}{2 \delta_{2}}+\frac{\theta_{M}^{2}}{2 \delta_{3}}\right)\left\|e_{c}^{j+1}-e_{c}^{j}\right\|^{2}+\tau \frac{M_{u}^{2}}{2 \delta_{4}}\left\|e_{c}^{j+1}\right\|^{2} \\
& +\tau \frac{\delta_{4}}{2}\left\|\nabla e_{c}^{j+1}\right\|^{2} .
\end{aligned}
$$

Adding (36) to (39) and using (A1), one gets

$$
\begin{aligned}
& \alpha_{\Psi}\left\|\theta_{h}^{n}-\theta_{h}^{n, j}\right\|^{2}+\frac{L_{1}}{2}\left\|e_{\Psi}^{j+1}\right\|^{2} \\
& +\frac{L_{1}}{2}\left\|e_{\Psi}^{j+1}-e_{\Psi}^{j}\right\|^{2}+\tau K_{m}\left\|\nabla e_{\Psi}^{j+1}\right\|^{2} \\
& +\alpha_{c}\left\|e_{c}^{j}\right\|^{2}+\theta_{m}\left\|e_{c}^{j+1}\right\|^{2}+\frac{L_{2}}{2}\left\|e_{c}^{j+1}\right\|^{2} \\
& +\frac{L_{2}}{2}\left\|e_{c}^{j+1}-e_{c}^{j}\right\|^{2}+\tau D\left\|\nabla e_{c}^{j+1}\right\|^{2} \\
& \leq \frac{L_{1}}{2}\left\|e_{\Psi}^{j}\right\|^{2}+\left(\frac{\delta_{0}}{2}+\frac{\tau\left(M_{\Psi}^{2}+1\right) L_{k}^{2}}{2 \delta_{1}}+\frac{\delta_{2}}{2}\right)\left\|\theta_{h}^{n, j}-\theta_{h}^{n}\right\|^{2} \\
& +\frac{1}{2 \delta_{0}}\left\|e_{\Psi}^{j+1}-e_{\Psi}^{j}\right\|^{2}+\tau \delta_{1}\left\|\nabla e_{\Psi}^{j+1}\right\|^{2}+\frac{L_{2}}{2}\left\|e_{c}^{j}\right\|^{2} \\
& +\frac{\delta_{3}}{2}\left\|e_{c}^{j}\right\|^{2}+\left(\frac{M_{c}^{2}}{2 \delta_{2}}+\frac{\theta_{M}^{2}}{2 \delta_{3}}\right)\left\|e_{c}^{j+1}-e_{c}^{j}\right\|^{2} \\
& +\tau \frac{M_{u}^{2}}{2 \delta_{4}}\left\|e_{c}^{j+1}\right\|^{2}+\tau \frac{\delta_{4}}{2}\left\|\nabla e_{c}^{j+1}\right\|^{2} \cdot
\end{aligned}
$$

Choosing $\delta_{0}=\delta_{2}=\frac{\alpha_{\Psi}}{2}, \delta_{1}=\frac{K_{m}}{2}, \delta_{3}=\theta_{m}$ and $\delta_{4}=\frac{D}{2}$ in (40), and assuming that

$$
L_{1} \geq \frac{2}{\alpha_{\Psi}} \text { and } L_{2} \geq \frac{2 M_{c}^{2}}{\alpha_{\Psi}}+\frac{\theta_{M}^{2}}{\theta_{m}}
$$

and the time step $\tau$ satisfies the mild conditions

$$
\begin{aligned}
& \alpha_{\Psi}-2 \tau \frac{\tau\left(M_{\Psi}^{2}+1\right) L_{k}^{2}}{K_{m}} \geq 0 \quad \text { and } \\
& \theta_{m}+2 \alpha_{c}+\frac{\tau D}{C_{\Omega}}-\frac{2 \tau M_{u}^{2}}{D} \geq 0,
\end{aligned}
$$

where $C_{\Omega}$ denotes the Poincare constant; then, we obtain

$$
\begin{aligned}
& \frac{L_{1}}{2}\left\|e_{\Psi}^{j+1}\right\|^{2}+\tau \frac{K_{m}}{2}\left\|\nabla e_{\Psi}^{j+1}\right\|^{2}+\left(\frac{L_{2}}{2}+\theta_{m}-\tau \frac{M_{u}^{2}}{D}\right) \\
& \times\left\|e_{c}^{j+1}\right\|^{2}+\tau \frac{D}{2}\left\|\nabla e_{c}^{j+1}\right\|^{2} \\
\leq & \frac{L_{1}}{2}\left\|e_{\Psi}^{j}\right\|^{2}+\left(\frac{L_{2}}{2}+\frac{\theta_{m}}{2}-\alpha_{c}\right)\left\|e_{c}^{j}\right\|^{2} .
\end{aligned}
$$

Finally, by using the Poincare inequality two times we get from (43)

$$
\begin{aligned}
& \left(L_{1}+\tau \frac{K_{m}}{C_{\Omega}}\right)\left\|e_{\Psi}^{j+1}\right\|^{2}+\left(L_{2}+2 \theta_{m}+\tau \frac{D}{C_{\Omega}}-2 \tau \frac{M_{u}^{2}}{D}\right) \\
& \times\left\|e_{c}^{j+1}\right\|^{2} \\
\leq & L_{1}\left\|e_{\Psi}^{j}\right\|^{2}+\left(L_{2}+\theta_{m}-2 \alpha_{c}\right)\left\|e_{c}^{j}\right\|^{2} .
\end{aligned}
$$

From (42), (44) implies that the errors are contracting and therefore the monolithic L-scheme (29)-(30) is convergent.

Remark 4 The convergence rate resulting from (44) does not depend on the spatial mesh size. Also, observe that this convergence is obtained for any initial guess. Based on this, the method is globally convergent, which is in contrast to the Newton and (modified) Picard schemes, converging only locally. It can be observed that, larger the time step and smaller constants $L_{1}$ and $L_{2}$, result in a faster convergence. For small steps instead the convergence rate can approach 1. On the other hand, if the time step is small enough, one may reach the regime where the Newton scheme becomes convergent (see [37]). Alternatively, one may first perform a number of L-scheme iterations, and use the resulting as an initial guess for the Newton scheme (see [30]), or consider the modified L-scheme in [31]. In either situations, the convergence behavior was much improved.

Remark 5 The convergence of the modified Picard and Newton method applied to the Richards equation has been already proved in [37]. Such results can be extended to the coupled problems considered here. 
Table 1 Parameters involved in all examples

\begin{tabular}{ll}
$L_{1}$ & 0.1 \\
$L_{2}$ & 0.1 \\
$D$ & $1 \mathrm{e}-3$ \\
Van Genuchten parameters & \\
$\theta_{s}$ & 0.42 \\
$\theta_{r}$ & 0.026 \\
$n$ & 2.9 \\
$\alpha$ & 0.551 \\
$a$ & 0.44 \\
$b$ & 0.0046 \\
$K_{s}$ & 0.12 \\
Accuracy requirement & \\
$\epsilon_{1}=\epsilon_{2}$ & $10^{-06}$ \\
\hline
\end{tabular}

\section{Numerical examples}

In this section we consider five test cases for the proposed linearization schemes, inspired by the literature [25, 30]. The schemes have been implemented in the open source software package MRST [29], an open source toolbox based on Matlab, in which multiple solvers and models regarding flows in porous media are incorporated.

\section{Example 1A: Flow and transport in a strictly unsaturated medium}

For the first example, a van Genuchten parametrization is considered, with the parameters given in Table 1. The domain $\Omega$ is the unit square. To define the initial pressure, $\Omega$ is divided into two sub-regions: $\Omega_{u p}=(0,1) \times$ $[1 / 4,1)$ and $\Omega_{\text {down }}=(0,1) \times(0,1 / 4)$. In $\Omega_{u p}$, the source term, in the Richards equation, is $H_{1}(x, y)=$ $0.06 \cos (4 / 3 \pi y) \sin (x)$ and $H_{2}(x, y)=0$, in the transport. The lower sub-domain $\Omega_{\text {down }}$ contains no external sources, i.e., $H_{1}=H_{2}=0$.
We impose $\Psi=-2$ on $\Gamma_{D}=[0,1] \times 1, c=1$ on $\Gamma_{D} \backslash \Gamma_{C}$, where $\Gamma_{C}=[1 / 3,2 / 3] \times 1, c=4$ on $\Gamma_{C}$ and no-flow Neumann boundary conditions for both model components on $\Gamma_{N}=\partial \Omega \backslash \Gamma_{D}$. At $t=0$ the initial pressure in the two sub-domains is $\Psi_{u p}^{0}=-2$ and $\Psi_{\text {down }}^{0}=$ $-y-1 / 4$, respectively. The initial concentration is $c^{0}=1$.

The simulations are performed on regular meshes, consisting of squares with sides $d x=1 / 10,1 / 20$, and $1 / 40$. The time steps are $\tau=1 / 10,1 / 20$, and 1/40. Figure 3 displays the pressure and concentration profiles at the final time $T=1$. Note that in this example the flow is always partially saturated, implying that the Richards equation does not degenerate. Furthermore, the flow is completely dominated by the source term $H_{1}$ while, for the transport, we can notice both diffusion and advection effects.

The total number of iterations and the condition numbers of the linear systems associated to each solving algorithms are presented in Tables 2 and 3. The condition numbers are computed using the $L^{1}$ norm and we report here the averaged values over the full simulation. A segment (-) in these tables implies that the method failed to converge for the particular combination of time step size and mesh. Table 2 gives the results obtained for the time step $\tau=$ $1 / 10$ and for different mesh sizes, $d x=1 / 10,1 / 20$ and $1 / 40$. In this case we can observe that the L-scheme based solvers converges for each mesh. The splitting solvers obtained instead thanks to the Newton and modified Picard linearizations, fail to converge in case of finer meshes. Furthermore, the numbers of iterations for the L-schemes is mesh independent, which agrees with the theory.

Similarly, Table 3 provides the results for a constant $d x=1 / 40$, and for $\tau=1 / 10,1 / 20$, and $1 / 40$. Observe that for the smaller time steps all schemes converge, whereas for $\tau=1 / 10$, the Newton and Picard-based variants of the splitting schemes diverge. This is in line with the results reported in Table 2, where a finer spatial mesh has led to the divergence of these schemes. Since the number of iterations is added per each time step, this number is
Fig. 3 Example 1A: pressure and concentration at the final time, $T=1$. The simulations were performed for $d x=1 / 100$ and $\tau=1 / 10$. a Pressure profile at $T=1$. b Concentration profile at $T=1$

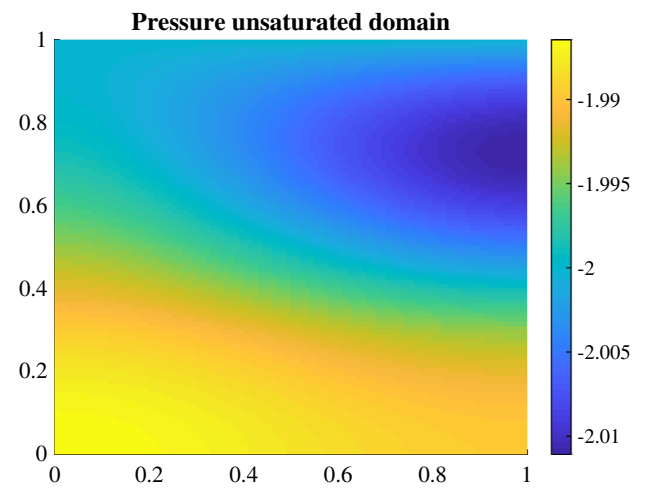

(a) Pressure profile at $\mathrm{T}=1$

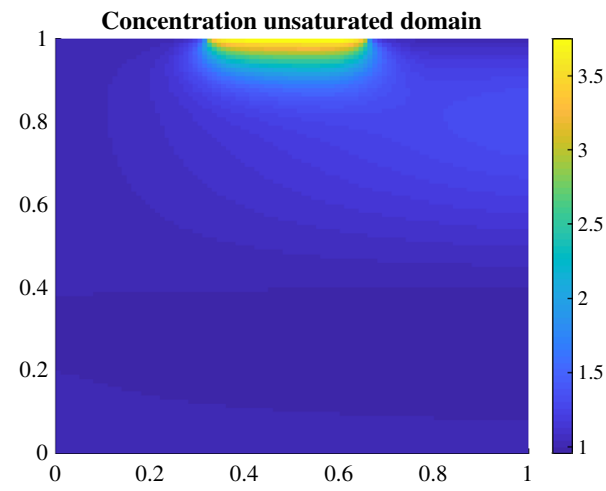

(b) Concentration profile at $\mathrm{T}=1$ 
Table 2 Example 1A: Iterations and condition numbers for fixed $\tau=1 / 10$

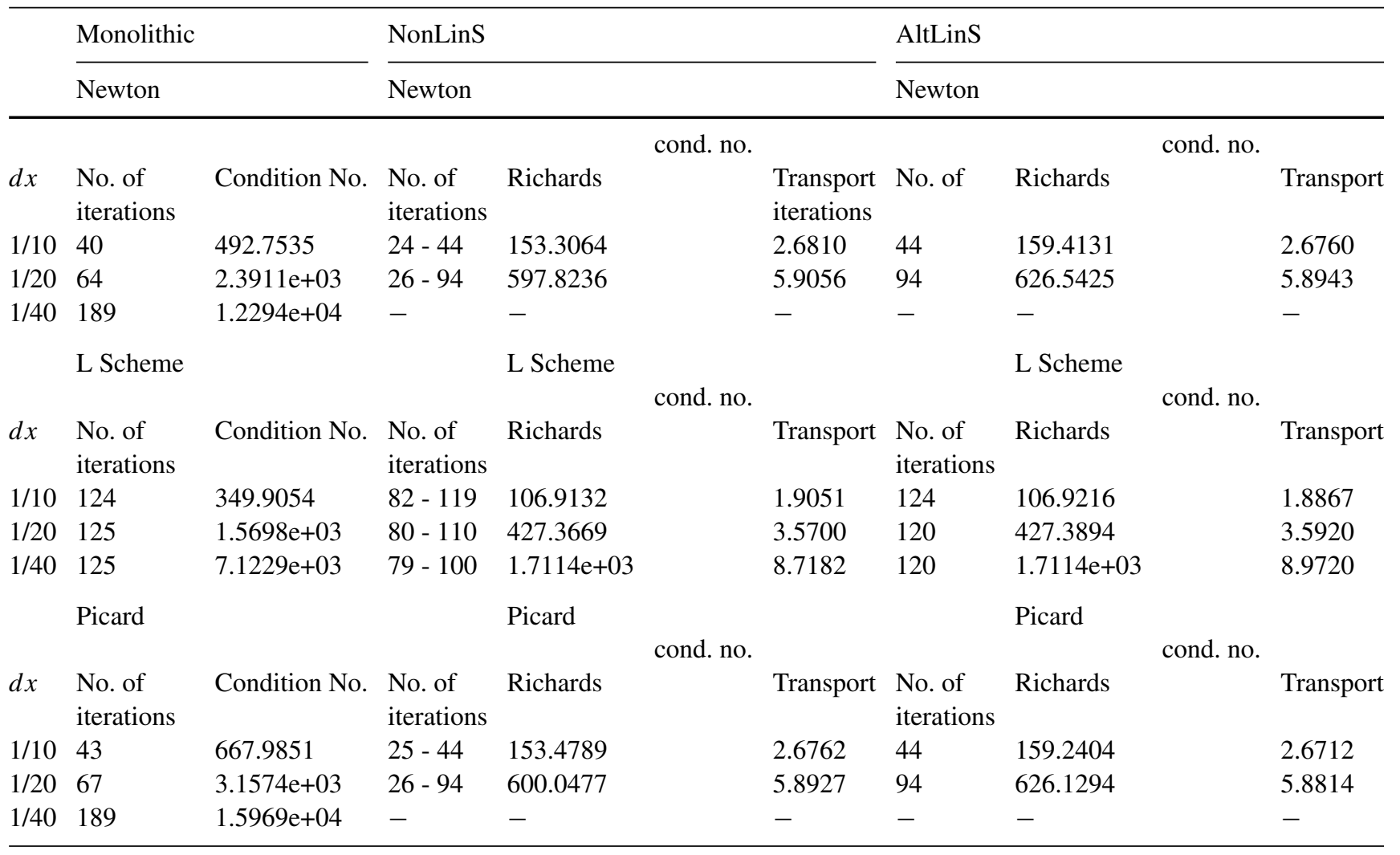

Table 3 Example 1A: Iterations and condition numbers for fixed $d x=1 / 40$

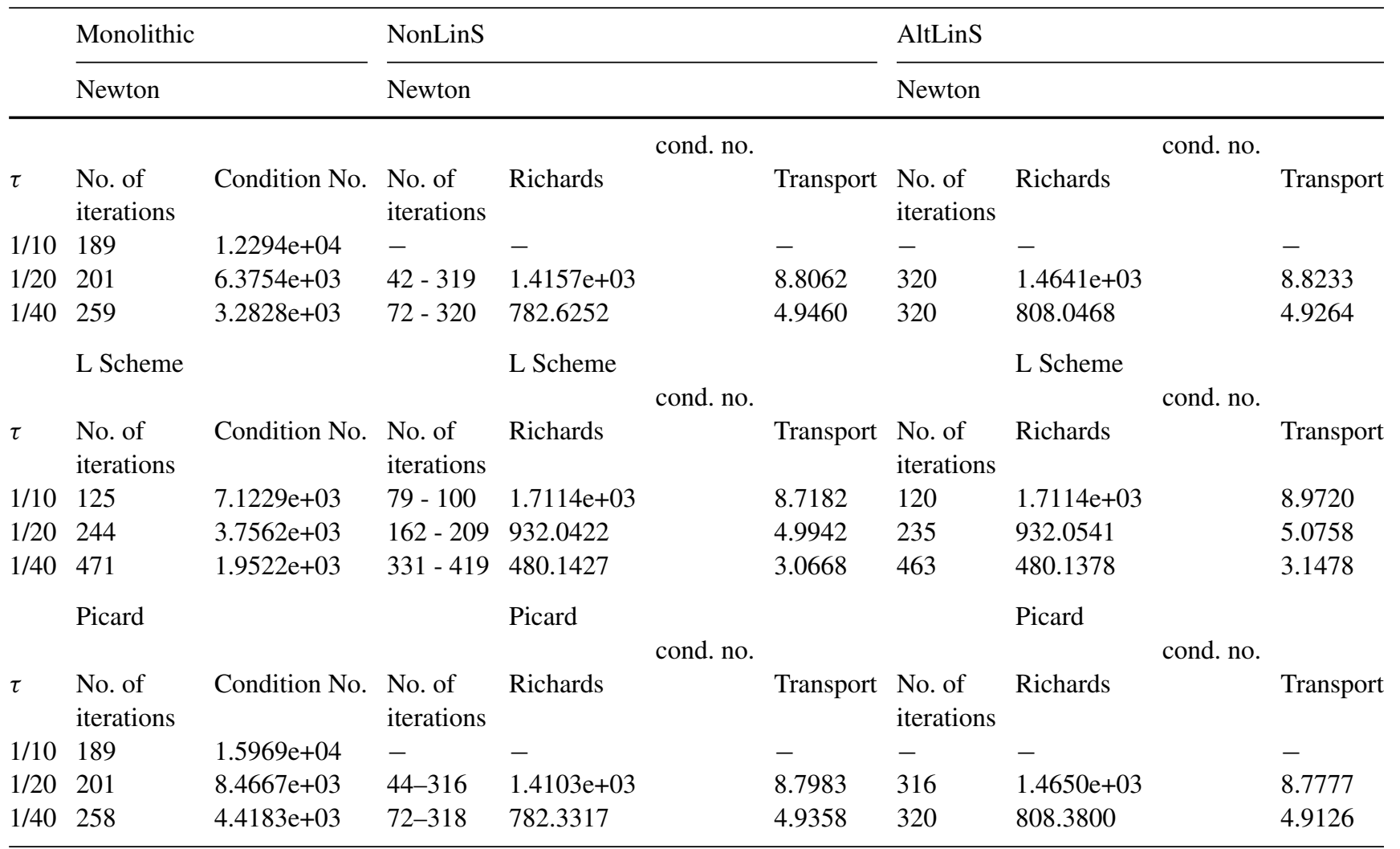


increasing as the time step is reduced. This is justified by the fact that smaller $\tau$ implies more time steps.

We point out that the alternate splitting schemes are converging much faster than the classical ones, for which we report the iterations required by the flow and the transport equations, separately. Note the differences in the condition numbers, the L-scheme-based algorithms being better conditioned. Observe also that for the splitting schemes, the condition numbers for the Richards equation are much larger than for the transport model component. This is due to the fact that the former is nonlinear and possibly degenerate, whereas the latter has a fairly simple structure. Finally, one can observe that finer meshes results in higher condition numbers while smaller time steps give better conditioned systems.

\section{Example 1B: Flow and transport in a variably saturated porous medium}

The situation given above is changed slightly, so that the fully saturated regime is achieved. Specially, we take $\Psi_{u p}^{0}=-2$ and $\Psi_{\text {down }}^{0}=-y+1 / 4$. By this, $\Psi_{\text {down }}^{0}$ becomes positive in $\Omega_{\text {down }}$, where the medium is fully saturated. Consequently, the Richards equation degenerates to an elliptic one, making the numerical simulation more challenging. The $L$ parameters are $L_{1}=L_{2}=0.2$.
Tables 4 and 5 present the iterations and condition numbers for each of the implemented algorithms, and for different mesh diameters and time steps. Note that in this case, only the L-scheme-based algorithms are converging. It is also interesting to observe the difference in the number of iterations between the more commonly used nonlinear splitting approach (NonLinS) and the alternate splitting (AltLinS) approach. The latter appears to be a valid alternative to the common formulation. It produces equally accurate results, requiring fewer iterations.

Finally, we observe as the Newton and Picard-based schemes fail to converge in all situations. This is due to the degeneracy of the Richards equation. The L-schemebased iterations did converge in all cases. The convergence behavior is as predicted by the theory: the number of iterations increases for smaller time steps, while the mesh size has no influence on the number of iterations.

\section{Example 2A: Well in an unsaturated porous medium}

The next example is inspired from [25]. We still consider the unit square-domain, the initial conditions and the parameters as in Example 1A. The medium results again strictly unsaturated. Now $\Omega_{u p}$ includes a well, and water with a given surfactant concentration is injected. The pressure at the well is set to $\Psi_{W}=-1 / 4$ and the

Table 4 Example 1B: Iterations and condition numbers for fixed $\tau=1 / 10$

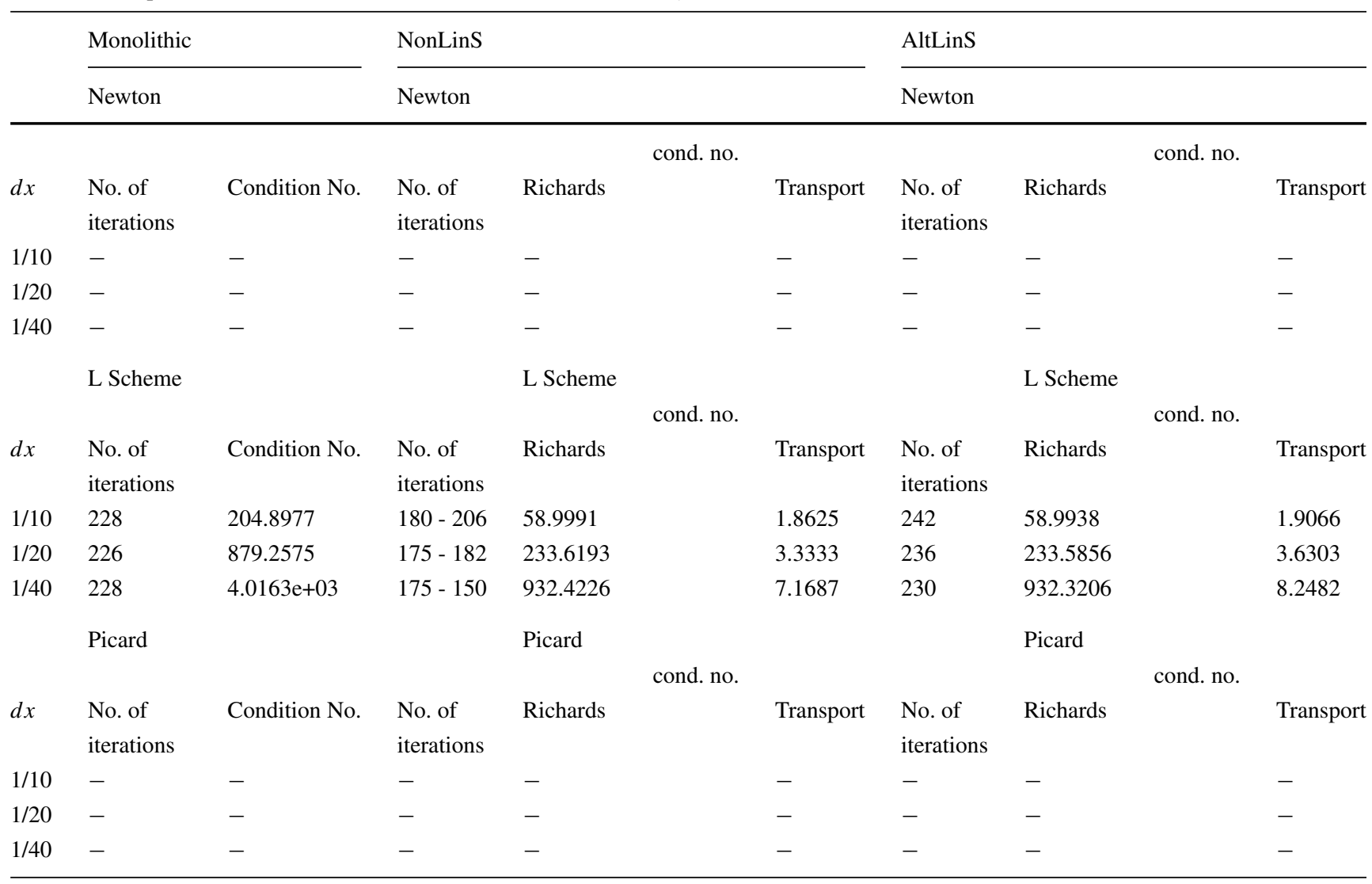


Table 5 Example 1B: Iterations and condition numbers for $d x=1 / 40$

\begin{tabular}{|c|c|c|c|c|c|c|c|c|c|}
\hline & Monolithic & & NonLinS & & & AltLinS & & & \\
\hline & Newton & & Newton & & & Newton & & & \\
\hline & & & & & & & & cond. no. & \\
\hline$\tau$ & $\begin{array}{l}\text { No. of } \\
\text { iterations }\end{array}$ & Condition No. & $\begin{array}{l}\text { No. of } \\
\text { iterations }\end{array}$ & Richards & Transport & $\begin{array}{l}\text { No. of } \\
\text { iterations }\end{array}$ & Richards & & Transport \\
\hline $1 / 10$ & - & - & - & - & - & - & - & & - \\
\hline $1 / 20$ & - & - & - & - & - & - & - & & - \\
\hline $1 / 40$ & - & - & - & - & - & - & - & & - \\
\hline & L Scheme & & & L Scheme & & & L Scheme & & \\
\hline$\tau$ & $\begin{array}{l}\text { No. of } \\
\text { iterations }\end{array}$ & Condition No. & $\begin{array}{l}\text { No. of } \\
\text { iterations }\end{array}$ & Richards & Transport & $\begin{array}{l}\text { No. of } \\
\text { iterations }\end{array}$ & Richards & cond. no. & Transport \\
\hline $1 / 10$ & 228 & $4.0163 e+03$ & $175-150$ & 932.4226 & 7.1687 & 230 & 932.3206 & & 8.2482 \\
\hline $1 / 20$ & 457 & $2.1063 \mathrm{e}+03$ & $362-326$ & 480.1123 & 4.2676 & 481 & 480.1165 & & 4.5628 \\
\hline $1 / 40$ & 877 & $1.0751 \mathrm{e}+03$ & $724-668$ & 241.9597 & 2.7304 & 944 & 241.9634 & & 2.7427 \\
\hline & Picard & & & Picard & & & Picard & & \\
\hline$\tau$ & $\begin{array}{l}\text { No. of } \\
\text { iterations }\end{array}$ & Condition No. & $\begin{array}{l}\text { No. of } \\
\text { iterations }\end{array}$ & Richards & Transport & $\begin{array}{l}\text { No. of } \\
\text { iterations }\end{array}$ & Richards & cond. no. & Transport \\
\hline $1 / 10$ & - & - & - & - & - & - & - & & - \\
\hline $1 / 20$ & - & - & - & - & - & - & - & & - \\
\hline $1 / 40$ & - & - & - & - & - & - & - & & - \\
\hline
\end{tabular}

concentration of the surfactant to $c_{W}=2$. We impose no-flow boundary conditions for both model components on $\partial \Omega$. The simulations are performed on regular meshes, consisting of squares with sides $d x=1 / 10,1 / 20$, and $1 / 40$. The time steps are $\tau=1 / 25,1 / 50$, and $1 / 100$. Furthermore, a reaction term is included in the transport equation, $R(c):=1 e-3 * c /(1+c)$. For the iteration $j+1$, this is linearized as $R\left(c^{n+1, j+1}\right) \rightarrow 1 e-3 \frac{c^{n+1, j+1}}{1+c^{n+1, j}}$. The $L$ parameters are $L_{1}=L_{2}=0.2$.
Figure 4 shows the pressure and the concentration at the final time step $(T=1)$. As for the first example, the medium being partially saturated, the Richards equation does not degenerate and almost all the schemes converge. The monolithic Newton method requires smaller time steps, as observable in Table 7. We remark, from both Tables 6 and 7 , that the alternate splitting approach (AltLinS), once more, requires fewer iterations than the classical splitting algorithm (NonLinS). The linear systems resulting by
Fig. 4 Example 2A: pressure and concentration at the first time steps and final times. The simulations were performed for $d x=1 / 80$ and $\tau=1 / 10$. a Pressure at final time $T_{1}$. b Concentration at final time $T_{1}$

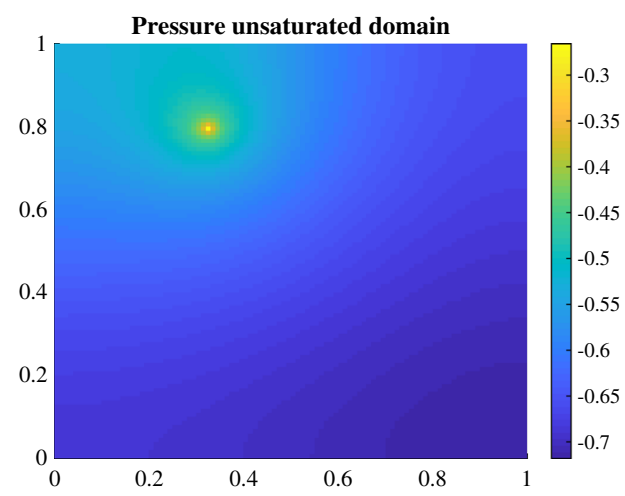

(a) Pressure at final time $T_{1}$

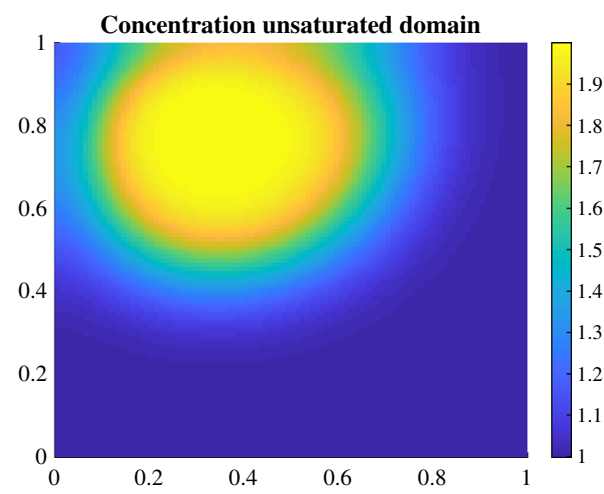

(b) Concentration at final time $T_{1}$ 
Table 6 Example 2A: Iterations and condition numbers for fixed $\tau=1 / 25$

\begin{tabular}{|c|c|c|c|c|c|c|c|c|c|c|}
\hline \multirow{3}{*}{ 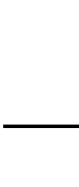 } & \multirow{2}{*}{\multicolumn{2}{|c|}{$\begin{array}{l}\text { Monolithic } \\
\text { Newton }\end{array}$}} & \multirow{2}{*}{\multicolumn{4}{|c|}{$\begin{array}{l}\text { NonLinS } \\
\text { Newton }\end{array}$}} & \multirow{2}{*}{\multicolumn{4}{|c|}{$\frac{\text { AltLinS }}{\text { Newton }}$}} \\
\hline & & & & & & & & & & \\
\hline & & & & & cond. no. & & & & cond. no. & \\
\hline$d x$ & $\begin{array}{l}\text { No. of } \\
\text { iterations }\end{array}$ & Condition No. & $\begin{array}{l}\text { No. of } \\
\text { iterations }\end{array}$ & Richards & & Transport & $\begin{array}{l}\text { No. of } \\
\text { iterations }\end{array}$ & Richards & & Transport \\
\hline $1 / 10$ & 59 & $1.1597 \mathrm{e}+03$ & $57-32$ & $3.5250 \mathrm{e}+03$ & & $1.6216 \mathrm{e}+05$ & - & - & & - \\
\hline $1 / 20$ & - & - & $57-35$ & $3.5250 \mathrm{e}+03$ & & $1.6216 \mathrm{e}+05$ & - & - & & - \\
\hline \multirow[t]{2}{*}{$1 / 40$} & - & - & $57-33$ & $3.9905 \mathrm{e}+04$ & & $2.4845 \mathrm{e}+06$ & - & - & & - \\
\hline & L Scheme & & & L Scheme & & & & L Scheme & & \\
\hline$d x$ & $\begin{array}{l}\text { No. of } \\
\text { iterations }\end{array}$ & Condition No. & $\begin{array}{l}\text { No. of } \\
\text { iterations }\end{array}$ & Richards & cond. no. & Transport & $\begin{array}{l}\text { No. of } \\
\text { iterations }\end{array}$ & Richards & cond. no. & Transport \\
\hline $1 / 10$ & 368 & $9.4574 \mathrm{e}+04$ & $209-315$ & 395.8757 & & $7.8808 \mathrm{e}+03$ & 338 & 397.4869 & & $7.9035 \mathrm{e}+03$ \\
\hline $1 / 20$ & 364 & $7.6020 \mathrm{e}+03$ & $222-327$ & $2.8139 \mathrm{e}+03$ & & $9.0509 e+04$ & 346 & $2.8220 \mathrm{e}+03$ & & $9.0971 \mathrm{e}+04$ \\
\hline \multirow[t]{2}{*}{$1 / 40$} & 368 & $9.4574 \mathrm{e}+04$ & $223-332$ & $3.0786 \mathrm{e}+04$ & & $1.3442 \mathrm{e}+06$ & 348 & $3.0861 \mathrm{e}+04$ & & $1.3515 \mathrm{e}+06$ \\
\hline & Picard & & & Picard & & & & Picard & & \\
\hline$\tau$ & $\begin{array}{l}\text { No. of } \\
\text { iterations }\end{array}$ & Condition No. & $\begin{array}{l}\text { No. of } \\
\text { iterations }\end{array}$ & Richards & cond. no. & Transport & $\begin{array}{l}\text { No. of } \\
\text { iterations }\end{array}$ & Richards & cond. no. & Transport \\
\hline $1 / 10$ & 106 & $2.1274 \mathrm{e}+03$ & $61-50$ & 426.0623 & & $1.2673 \mathrm{e}+04$ & 74 & 416.8809 & & $1.2985 \mathrm{e}+04$ \\
\hline $1 / 20$ & 105 & $2.1056 \mathrm{e}+04$ & $70-50$ & $3.4855 \mathrm{e}+03$ & & $1.5665 \mathrm{e}+05$ & 84 & $3.3929 \mathrm{e}+03$ & & $1.6001 \mathrm{e}+05$ \\
\hline $1 / 40$ & 105 & $2.7185 e+05$ & $84-50$ & $3.9872 \mathrm{e}+04$ & & $2.3954 \mathrm{e}+06$ & 86 & $3.9462 \mathrm{e}+04$ & & $2.4544 \mathrm{e}+06$ \\
\hline
\end{tabular}

Table 7 Example 2A: Iterations and condition numbers for $d x=1 / 20$

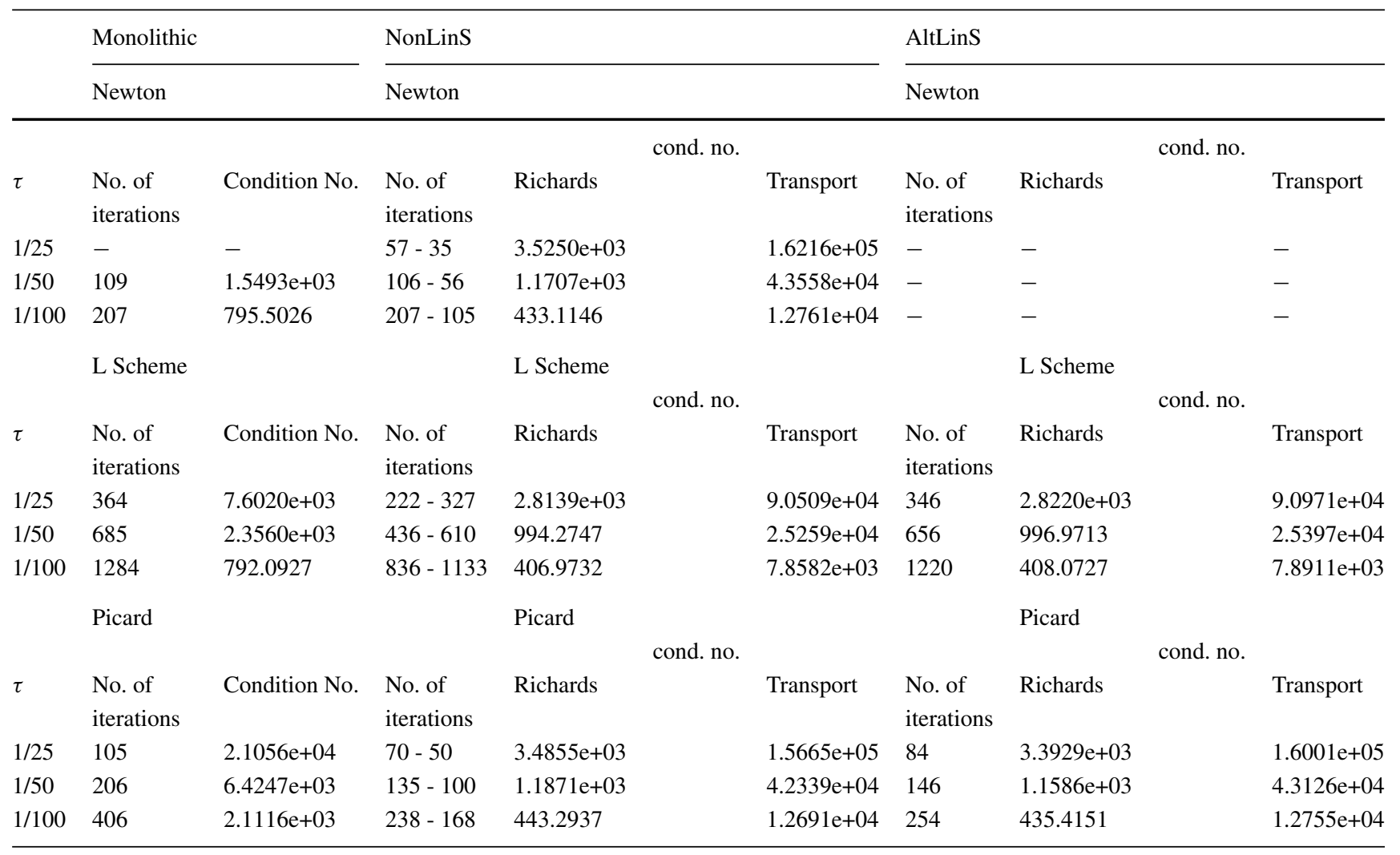


Table 8 Example 2B: Iterations and condition numbers for $\tau=1 / 25$

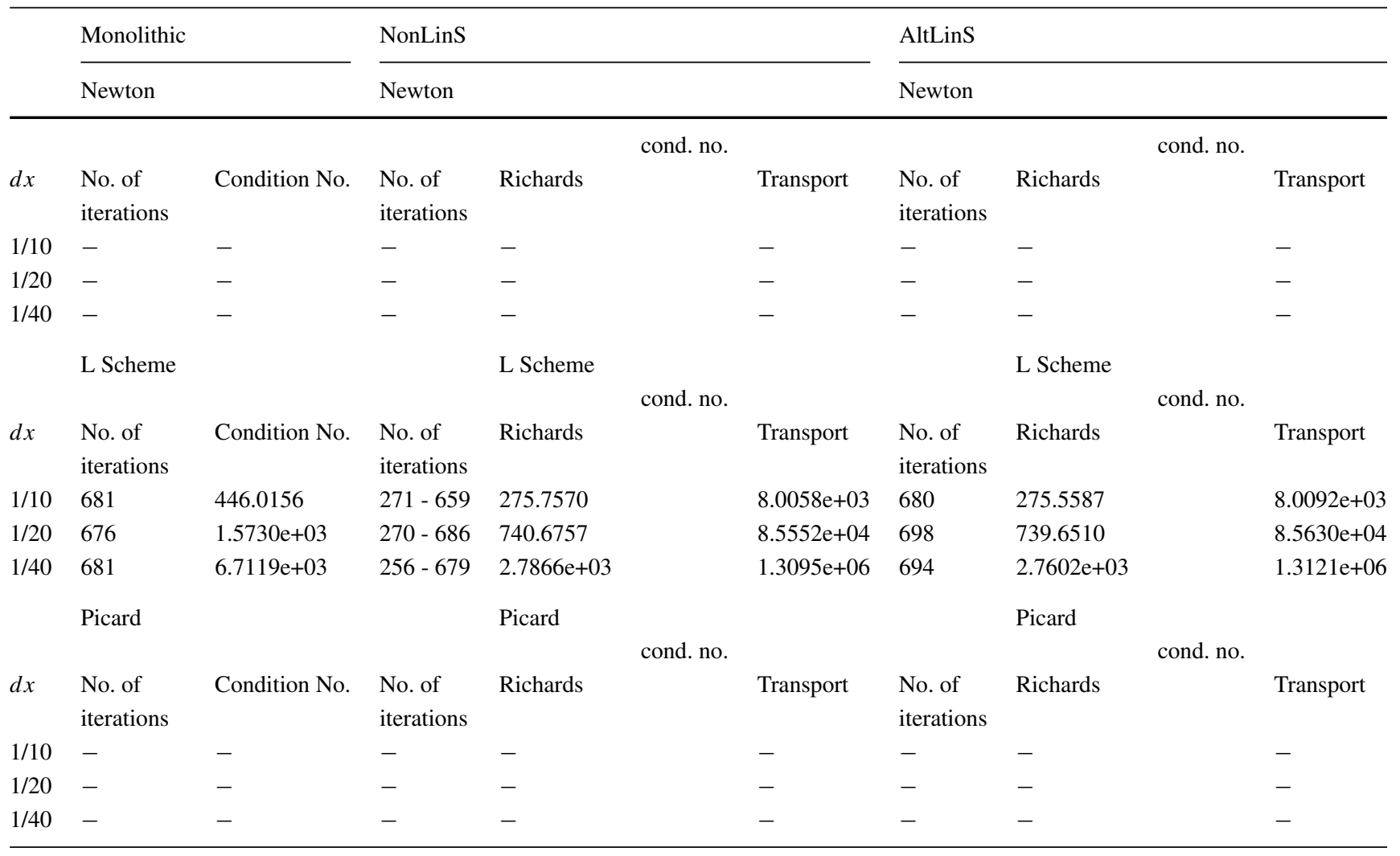

Table 9 Example 2B: Iterations and condition numbers for $d x=1 / 20$

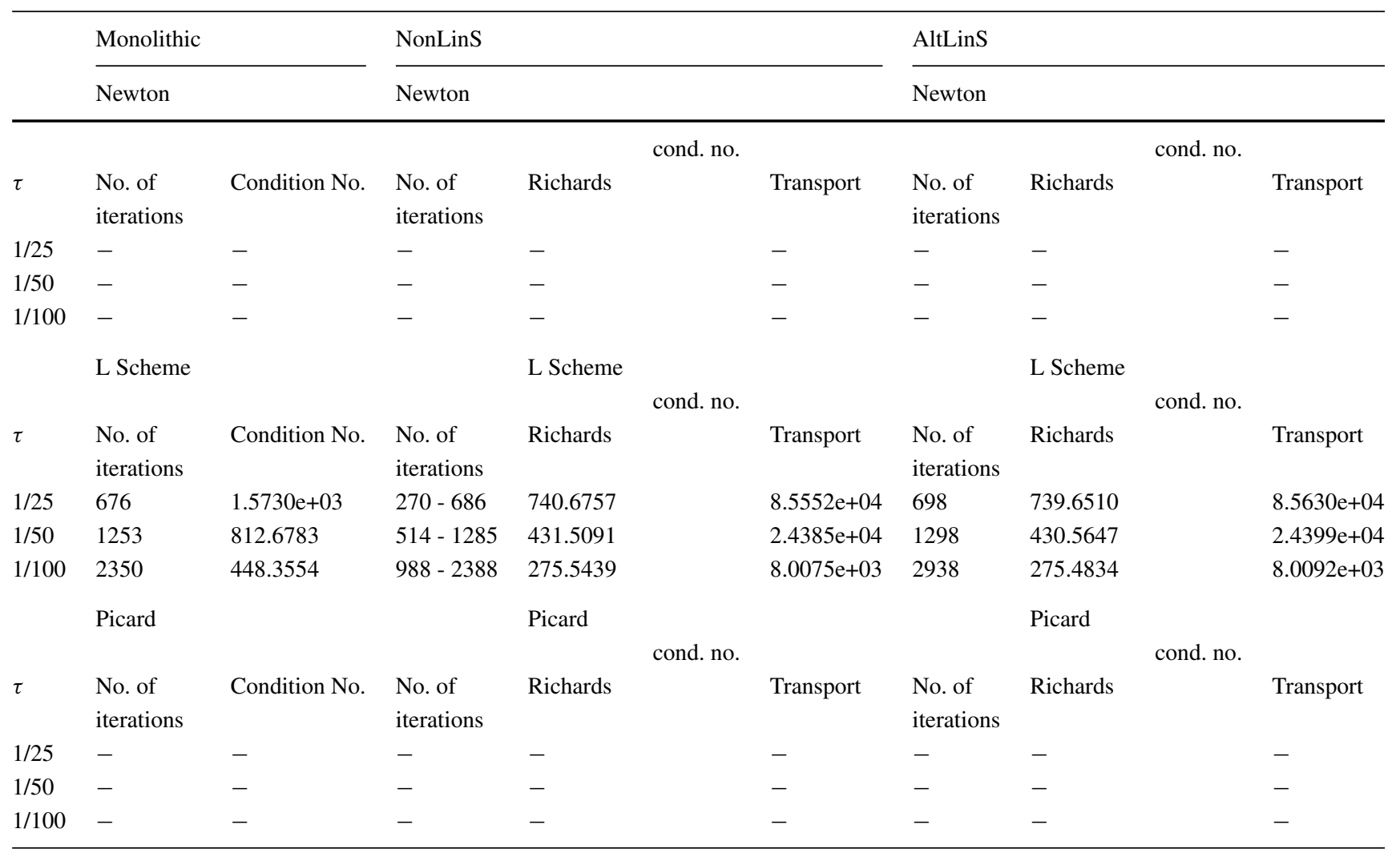


Fig. 5 Example 3: A highly heterogeneous domain. a Porosity of the domain $\Omega$. b Permeability of the domain $\Omega$

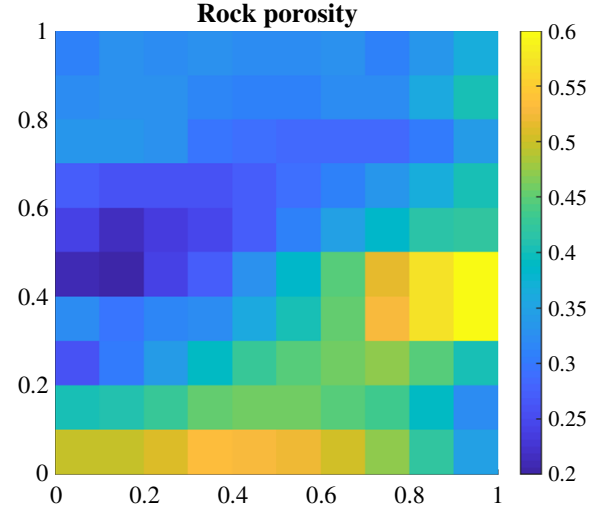

(a) Porosity of the domain $\Omega$

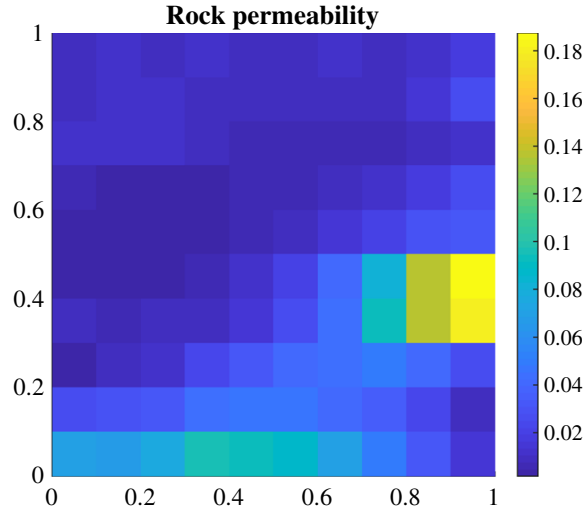

(b) Permeability of the domain $\Omega$ applying the L-scheme-based solvers are better conditioned compared to the other solvers. Finally, we can observe as the introduction of a nonlinear reaction term has drastically increased the condition numbers of the system associated to the transport equation.

\section{Example 2B: Well in a variably saturated porous medium}

As in Example 1B, now the initial condition for the pressure is changed, leading to a variably saturated porous media. The pressure at the well is fixed equal to $1 / 4$ and the concentration and boundary conditions are defined as in the Example 2A. The $L$ parameters are now $L_{1}=0.2$ and $L_{2}=0.5$.

As for Example 1B, since the Richards equation degenerates, many of the considered schemes show convergence problems. Tables 8 and 9 present the convergence of the schemes and the condition numbers for the associated linear systems. The results are very similar to those in the previous examples, with the L-scheme-based solvers being the most robust ones and the only converging for all cases. Furthermore, the alternate method is faster than the classical splitting scheme.

\section{Example 3: A heterogeneous porous medium}

In more realistic situations, the porous medium is often heterogeneous. In this example we consider again the unitsquare domain but with highly heterogeneous properties (porosity and permeability), as presented in Fig. 5. Next to this, the problem is similar to the one in Example 2B, including the same initial conditions and parameters. The well is now located in the lower right part of the domain where we observe larger porosity and permeability. Due to the initial pressure $\Psi^{0}$, the domain results to be variably saturated and the problem degenerates. The $L$ parameters are $L_{1}=L_{2}=0.7$.

Figure 6 present the pressure and concentration at the final time step. We can observe as, particularly the former, has increased in the regions with higher permeability. Similarly we can observe how the structure of the media has influence the diffusion of the external component.

Tables 10 and 11 present the total numbers of iterations for each algorithm and the condition numbers of the associated linearized systems. For this particular problem, it is interesting to notice that the L-scheme converges, again, for every time step and mesh investigated. In this
Fig. 6 Example 3: Pressure and concentration at the final time. The simulations are performed for $d x=1 / 40$ and $\tau=d x / 10$. a Pressure at the final time $T . \mathrm{b}$ Concentration at the final time $T$

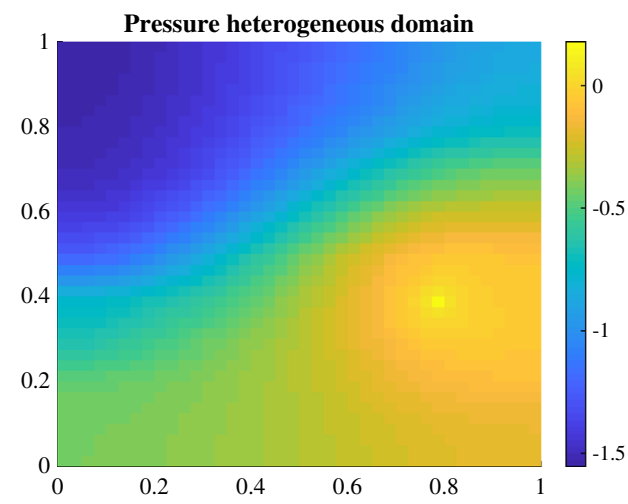

(a) Pressure at the final time $T$

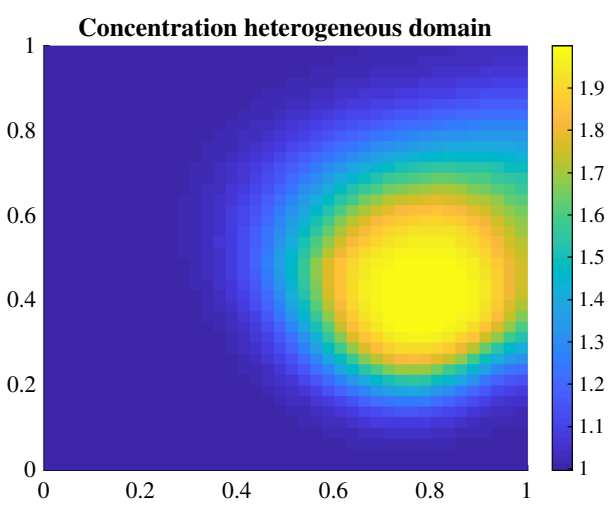

(b) Concentration at the final time $T$ 
Table 10 Example 3: Iterations and condition numbers for $\tau=1 / 10$

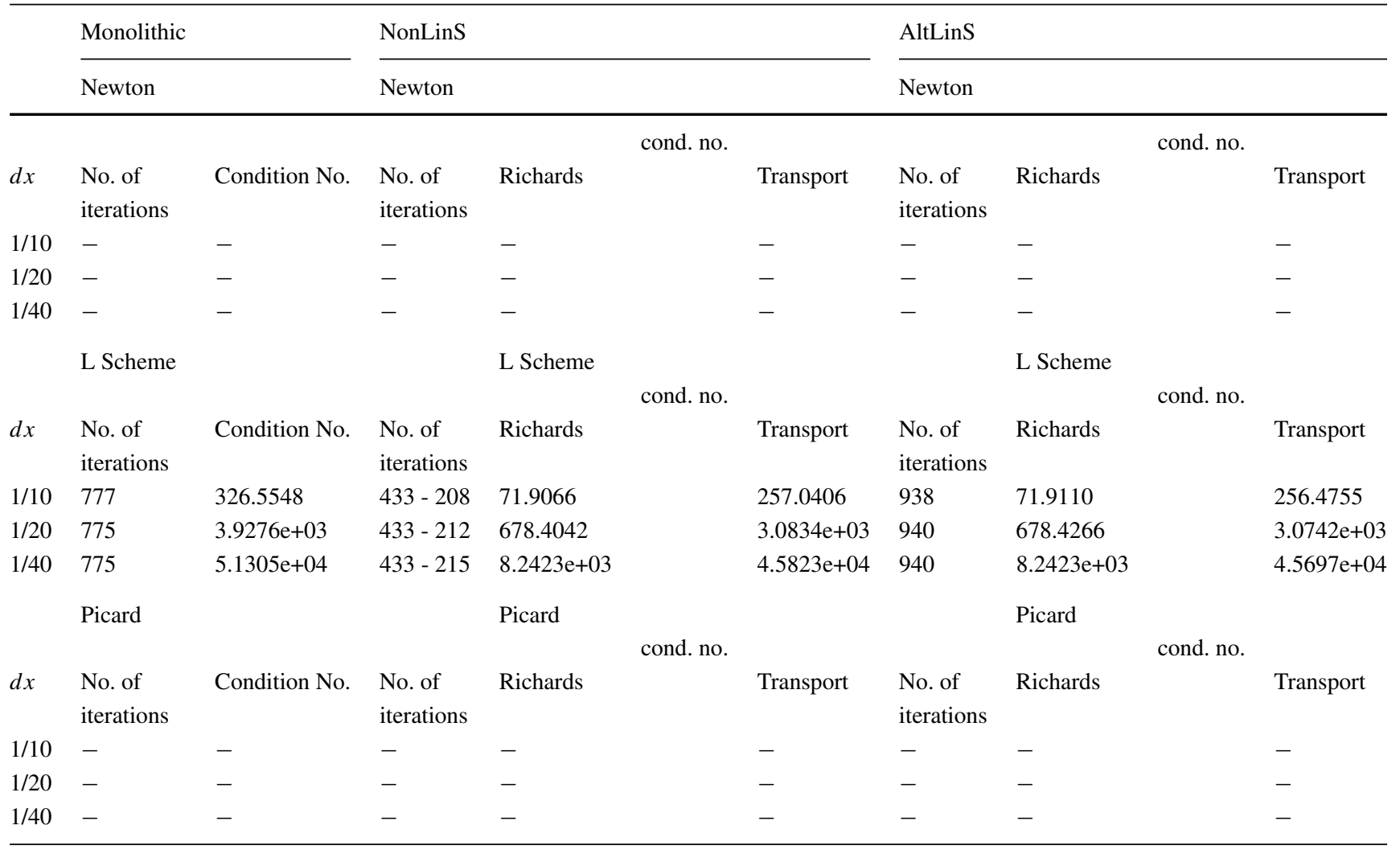

Table 11 Example 3: iterations and condition numbers for $d x=1 / 10$

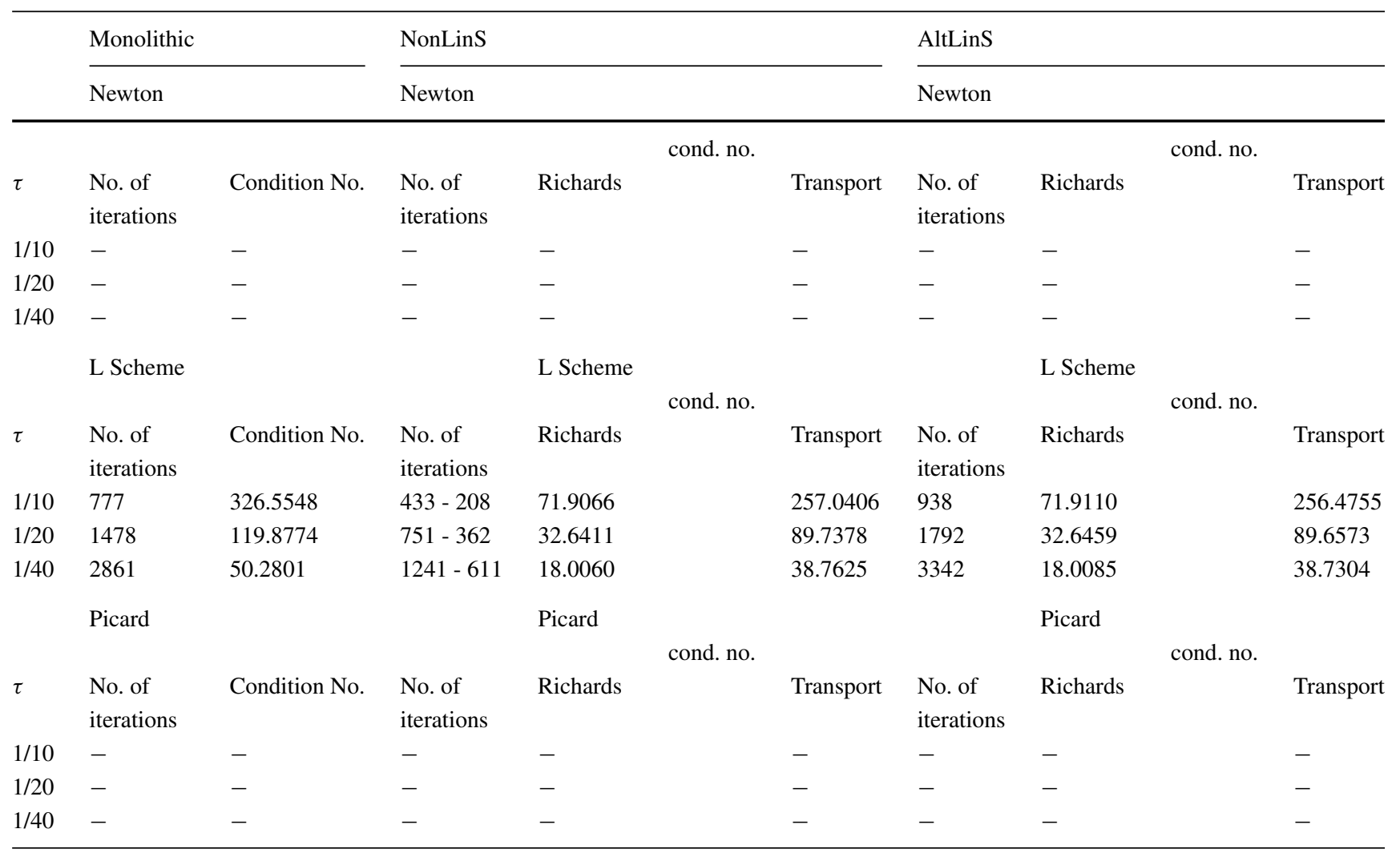


case, neither the Newton method nor the modified Picard, converged, even when smaller time steps were investigated. Smaller values were not tested because the resulting number of iterations would have been much larger than the one obtained with the original $\tau$ and the L-scheme. These results are coherent with the ones previously investigated. Whenever the Richards equation degenerate, both Newton and modified Picard present convergence problems.

\section{Conclusions}

In this paper, we studied different algorithms for the numerical solution of a surfactant transport model in variably saturated porous media. The water flow and the transport are fully coupled. Three linearization techniques were considered: the Newton method, the modified Picard and the L-scheme. Based on these, monolithic and splitting schemes were designed, analyzed and tested numerically. We conclude that the only quadratic convergent scheme is the monolithic Newton, that the L-scheme-based solvers are the most robust ones and produce well-conditioned linear systems and that the alternative schemes are often faster than the classical splitting approaches.

Although acknowledging the existence of improved Newton solvers, having a more robust convergence behavior, the present study shows that the L-scheme is a viable alternative. It can be particularly useful in the degenerate cases, or whenever large time steps have to be considered.

Acknowledgments Open Access funding provided by University of Bergen. We thank the members of the Sintef research group and in particular to Dr. Olav Moyner for the assistance with the implementation of the numerical examples in MRST, the toolbox based on MATLAB developed at Sintef itself.

Funding information The research of D. Illiano was funded by VISTA, a collaboration between the Norwegian Academy of Science and Letters and Equinor, project number 6367, project name: adaptive model and solver simulation of enhanced oil recovery. The research of I.S. Pop was supported by the Research Foundation-Flanders (FWO), Belgium, through the Odysseus programme (project G0G1316N) and Equinor through the Akademia grant.

Open Access This article is licensed under a Creative Commons Attribution 4.0 International License, which permits use, sharing, adaptation, distribution and reproduction in any medium or format, as long as you give appropriate credit to the original author(s) and the source, provide a link to the Creative Commons licence, and indicate if changes were made. The images or other third party material in this article are included in the article's Creative Commons licence, unless indicated otherwise in a credit line to the material. If material is not included in the article's Creative Commons licence and your intended use is not permitted by statutory regulation or exceeds the permitted use, you will need to obtain permission directly from the copyright holder. To view a copy of this licence, visit http:// creativecommonshorg/licenses/by/4.0/.

\section{References}

1. Aavatsmark, I.: An introduction to multipoint flux approximations for quadrilateral grids. Comput. Geosci. 6(3-4), 405-432 (2002)

2. Agosti, A., Formaggia, L., Scotti, A.: Analysis of a model for precipitation and dissolution coupled with a Darcy flux. J. Math. Anal. Appl. 431(2), 752-781 (2015)

3. Alt, W., Luckhaus, H.: Quasilinear elliptic-parabolic differential equations. Math. Z. 183(3), 311-341 (1983)

4. Arbogast, T., Wheeler, M.F.: A nonlinear mixed finite element method for a degenerate parabolic equation arising in flow in porous media. SIAM J. Numer. Anal. 33(4), 1669-1687 (1996)

5. Barrett, J.W., Knabner, P.: Finite element approximation of the transport of reactive solutes in porous media. Part 1: error estimates for nonequilibrium adsorption processes. SIAM J. Numer. Anal. 34(1), 201-227 (1997)

6. Bause, M., Hoffmann, J., Knabner, P.: First-order convergence of multi-point flux approximation on triangular grids and comparison with mixed finite element methods. Numer. Math. 116(1), 1-29 (2010)

7. Berardi, M., Difonzo, F., Vurro, M., Lopez, L.: The 1D Richards' equation in two layered soils: a Filippov approach to treat discontinuities. Adv. Water Resour. 115, 264-272 (2018)

8. Bergamaschi, L., Putti, M.: Mixed finite elements and Newtontype linearizations for the solution of Richards' equation. Int. J. Numer. Methods Eng. 45(8), 1025-1046 (1999)

9. Cances, C., Pop, I.S., Vohralik, M.: An a posteriori error estimate for vertex-centered finite volume discretizations of immiscible incompressible two-phase flow. Math. Comput. 83, 153-188 (2014)

10. Celia, M., Bouloutas, E., Zarba, R.L.: A General MassConservative Numerical Solution for the Unsaturated Flow Equation. Adv. Water Resour. 26(7), 1483-1496 (1990)

11. Christofi, N., Ivshina, I.B.: Microbial surfactants and their use in field studies of soil remediation. J. Appl. Microbiol. 93(6), 915-929 (2002)

12. Dawson, C.: Analysis of an upwind-mixed finite element method for nonlinear contaminant transport equations. SIAM J. Numer. Anal. 35(5), 1709-1724 (1998)

13. Eymard, R., Gutnic, M., Hilhorst, D.: The finite volume method for Richards equation. Comput. Geosci. 3(3-4), 256-294 (1999)

14. Eymard, R., Hilhorst, D., Vohral, M.: A combined finite volumenonconforming/mixed-hybrid finite element scheme for degenerate parabolic problems. Numer. Math. 105(1), 73-131 (2006)

15. Farthing, M.W., Ogden, F.L.: Numerical solution of Richards' equation: A review of advances and challenges. Soil Sci. Soc. Am. J. 81, 1257-1269 (2017)

16. Gallo, C., Manzini, G., mixed finite, A.: element/finite volume approach for solving biodegradation transport in groundwater. Internal Journal for Numerical Methods in Fluids 26(5), 533-556 (1998)

17. van Genuchten, M.: A closed-form equation for predicting the hydraulic conductivity of unsaturated soils. Soil Sci. Soc. Am. J. 44(5), 892-898 (1980)

18. Helmig, R.: Multiphase flow and transport processes in the subsurface: A contribution to the modeling of hydrosystems. Springer-Verlag, Berlin (1997)

19. Henry, E.J., Smith, J.E., Warrick, A.W.: Solubility effects on surfactant-induced unsaturated flow through porous media. J Hydrol 223(3-4), 164-174 (1999)

20. Husseini, D.: Effects of anions acids on surface tension of water, Undergraduate Research at JMU Scholarly Commons (2015) 
21. Jones, J.E., Woodward, C.S.: Newton-Krylov-multigrid solvers for large-scale, highly heterogeneous, variably saturated flow problems. Adv. Water Resour. 24(7), 763-774 (2001)

22. Jenny, P., Tchelepi, H.A., Lee, S.H.: Unconditionally convergent nonlinear solver for hyperbolic conservation laws with S-shaped flux functions. J. Comput. Phys. 228(20), 7497-7512 (2009)

23. Karagunduz, A., Young, M.H., Pennell, K.D.: Influence of surfactants on unsaturated water flow and solute transport. Water Resour. Res. 51(4), 1977-1988 (2015)

24. Klausen, R.A., Radu, F.A., Eigestad, G.T.: Convergence of MPFA on triangulations and for Richards' equation. Int. J. Numer. Methods Fluids 58(12), 1327-1351 (2008)

25. Knabner, P., Bitterlich, S., Teran, R.I., Prechtel, A., Schneid, E.: Influence of surfactants on spreading of contaminants and soil remediation. Springer, Berlin (2003)

26. Knoll, D.A., Keyes, D.E.: Jacobian-free Newton-Krylov methods: A survey of approaches and applications. J. Comput. Phys. 193(2), 357-397 (2004)

27. Kumar, K., Pop, I.S., Radu, F.A.: Convergence analysis of mixed numerical schemes for reactive flow in a porous medium. SIAM J. Numer. Anal. 51(4), 2283-2308 (2013)

28. Lee, S.H., Efendiev, Y.: C1-Continuous relative permeability and hybrid upwind discretization of three phase flow in porous media. Adv. Water Resour. 96, 209-224 (2016)

29. Lie, K.-A.: An introduction to reservoir simulation using MATLAB: User guide for the Matlab reservoir simulation toolbox (MRST), SINTEF ICT (2016)

30. List, F., Radu, F.A.: A study on iterative methods for solving Richards' equation. Comput. Geosci. 20(2), 341-353 (2016)

31. Mitra, K., Pop, I.S.: A modified L-Scheme to solve nonlinear diffusion problems. Comput. Math. Appl. 77, 1722-1738 (2019)

32. Nochetto, R., Verdi, C.: Approximation of degenerate parabolic problems using numerical integration. SIAM J. Numer. Anal. 25, 784-814 (1988)

33. Prechtel, A., Knabner, P.: Accurate and efficient simulation of coupled water flow and nonlinear reactive transport in the saturated and vadose zone - application to surfactant enhanced and intrinsic bioremediation. Int. J. Water Res. Dev. 47, 687-694 (2002)

34. Pop, I.S., Radu, F.A., Knabner, P.: Mixed finite elements for the Richards' equation: linearization procedure. J. Comput. Appl. Math. 168(1), 365-373 (2004)

35. Radu, F.A., Pop, I.S., Attinger, S.: Analysis of an Euler implicit, mixed finite element scheme for reactive solute transport in porous media. Num. Methods Part. Diff. Equ. 26(2), 320-344 (2010)

36. Radu, F.A., Pop, I.S., Knabner, P.: Order of convergence estimates for an Euler implicit, mixed finite element discretization of
Richards' equation. SIAM J. Numer. Anal. 42(4), 1452-1478 (2004)

37. Radu, F.A., Pop, I.S., Knabner, P.: On the convergence of the Newton method for the mixed finite element discretization of a class of degenerate parabolic equation, Numerical Mathematics and Advanced Applications, pp. 1192-1200 (2006)

38. Radu, F.A., Suciu, N., Hoffmann, J., Vogel, A., Kolditz, O., Park, C.H., Attinger, S.: Accuracy of numerical simulations of contaminant transport in heterogeneous aquifers: a comparative study. Adv. Water Resour. 34(1), 47-61 (2011)

39. Russell, T.F., Wheeler, M.F.: Finite element and finite difference methods for continuous flows in porous media, SIAM, pp. 35-106 (1983)

40. Slodicka, M.: A robust and efficient linearization scheme for doubly non-linear and degenerate parabolic problems arising in flow in porous media. SIAM J. Numer. Anal. 23(5), 1593-1614 (2002)

41. Smith, J.E., Gillham, R.W.: The effect of concentration-dependent surface tension on the flow of water and transport of dissolved organic compounds: A pressure head-based formulation and numerical model. Water Resour. Res. 31(3), 343-354 (1994)

42. Smith, J., Gillham, R.: Effects of solute concentration-dependent surface tension on unsaturated flow: Laboratory sand column experiments. Water Res. Res. 35(4), 973-982 (1999)

43. Suciu, N.: Diffusion in random velocity fields with applications to contaminant transport in groundwater. Water Res. Res. 69, 114-133 (2014)

44. Vohralik, M.: A posteriori error estimates for lowest-order mixed finite element discretizations of convection-diffusion-reaction equations. SIAM J. Numer. Anal. 45(4), 1570-1599 (2007)

45. Walker, H.F., Ni, P.: Anderson acceleration for fixed-point iterations. SIAM J. Numer. Anal. 49(4), 1715-1735 (2011)

46. Wang, X., Tchelepi, H.A.: Trust-region based solver for nonlinear transport in heterogeneous porous media. J. Comput. Phys. 253, 114-137 (2013)

47. Woodward, C.S., Dawson, C.N.: Analysis of expanded mixed finite element methods for a nonlinear parabolic equation modeling flow into variably saturated porous media. SIAM J. Numer. Anal. 37(3), 701-724 (2000)

48. Yong, W.A., Pop, I.S.: A numerical approach to porous medium equations, Preprint 95-50 (SFB 359), IWR University of Heidelberg (1996)

49. Younis, R., Tchelepi, H.A., Aziz, K.: Adaptively localized continuation-newton method-nonlinear solvers that converge all the time. SPE J. 15(02), 526-544 (2010)

Publisher's note Springer Nature remains neutral with regard to jurisdictional claims in published maps and institutional affiliations. 